\title{
Highly Ordered Smectic Phases from Polar Calix[4]arene Derivatives
}

\author{
Pik Kwan Lo ${ }^{a}$, Dongzhong Chen ${ }^{\mathrm{b}, *}$, Qingwei Meng ${ }^{\mathrm{b}}$, Man Shing Wong ${ }^{\mathrm{a}, *}$ \\ ${ }^{a}$ Department of Chemistry, Hong Kong Baptist University, Kowloon Tong, Hong Kong, \\ S.A.R. China. E-mail: mswong@hkbu.edu.hk \\ ${ }^{b}$ Key Laboratory of Mesoscopic Chemistry of Ministry of Education and Department of \\ Polymer Science and Engineering, School of Chemistry and Chemical Engineering, \\ Nanjing University, Nanjing 210093. China. E-mail: cdz@nju.edu.cn
}

\section{Contents}

I. Synthetic procedure and spectral data of calix[4]arene derivatives and monomers

II. Summaries of DSC results for calix[4]arene derivatives and monomers

III. Some representative POM textures for calix[4]arene derivatives and monomers

IV. XRD experimental result 


\section{Expermental Procedure:}

${ }^{1}$ H NMR spectra were recorded using a Jeol JHM-EX270 FT NMR spectrometer or a Varian INOVA-400 FT NMR spectrometer and are referenced to the residual $\mathrm{CHCl}_{3} 7.24 \mathrm{ppm}$ or DMSO 2.50 ppm. ${ }^{13} \mathrm{C}$ NMR spectra were recorded using a Jeol JHM-EX270 FT NMR spectrometer or a Varian INOVA-400 FT NMR spectrometer and are referenced to the $\mathrm{CDCl}_{3} 77$ ppm or DMSO-D 39.5 ppm. Mass spectroscopy (MS) measurements were carried using either fast atom bombardment (FAB) or matrix-assisted laser desorption ionizationtime-of-flight (MALDI-TOF) technique.

5,11,17,23-Tetrakisiodo-25,26,27,28-tetraoctyloxycalix[4]arene 3a. The alkylation procedure was followed using calix[4]arene $(0.26 \mathrm{~g}, 0.6 \mathrm{mmol})$ and 1-bromooctane $(0.64 \mathrm{~mL}, 5.4 \mathrm{mmol})$. The crude product was filtered by short silica-gel column chromatography using petroleum ether $/ \mathrm{CH}_{2} \mathrm{Cl}_{2}$ (v/v 8:1) as eluent affording (25,26,27,28-tetraoctyloxycalix[4]arene) as a viscous liquid. Following the above iodination procedure using 25,26,27,28-tetraoctyloxycalix[4]arene $(0.72 \mathrm{~g}, 0.8 \mathrm{mmol})$ in $40 \mathrm{~mL} \mathrm{CHCl}_{3}$, silver trifluoroacetate $(1.09 \mathrm{~g}, 4.9 \mathrm{mmol})$ and iodine $(1.29 \mathrm{~g}$, $5.1 \mathrm{mmol})$. The pure product was separated by silica-gel column chromatography using petroleum ether/ $\mathrm{CH}_{2} \mathrm{Cl}_{2}$ (v/v 8:1) as eluent affording $0.68 \mathrm{~g}(60 \%)$ of a viscous liquid. ${ }^{1} \mathrm{H}$ NMR $\left(400 \mathrm{MHz}, \mathrm{CDCl}_{3}, \delta\right) 7.01(\mathrm{~m}, 8 \mathrm{H}), 4.29(\mathrm{~d}, J=13.20 \mathrm{~Hz}, 4 \mathrm{H}), 3.85(\mathrm{t}, J=7.60 \mathrm{~Hz}, 8 \mathrm{H}), 3.05(\mathrm{~d}$, $J=14.80 \mathrm{~Hz}, 4 \mathrm{H}), 1.87(\mathrm{~m}, 8 \mathrm{H}), 1.33(\mathrm{bs}, 40 \mathrm{H}), 0.88(\mathrm{t}, J=6.75 \mathrm{~Hz}, 12 \mathrm{H}) .{ }^{13} \mathrm{C} \mathrm{NMR}(100$ $\left.\mathrm{MHz}, \mathrm{CDCl}_{3}, \delta\right) 156.2,136.9,136.6,86.1,31.9,30.3,30.1,29.8,29.5,26.1,22.6,14.0 . \quad \mathrm{MS}$ (FAB) $m / z 1376.2\left(\mathrm{M}^{+}\right)$.

\section{5,11,17,23-Tetrakisiodo-25,26,27,28-tetranonyloxycalix[4]arene $3 b . \quad$ The alkylation} procedures were followed using 25,26,27,28-tetra- hydroxycalix[4]arene (1.24 g, $2.92 \mathrm{mmol})$ and 1-bromononane $(3.62 \mathrm{~mL}, 17.4 \mathrm{mmol})$. The crude product was filtered by short silica-gel 
column chromatography using petroleum ether $/ \mathrm{CH}_{2} \mathrm{Cl}_{2}$ (v/v 8:1) as eluent affording $(25,26,27,28$-tetra-nonyloxycalix[4]arene) as a viscous liquid. Following the above iodination procedure using 25,26,27,28-tetranonyloxycalix[4]arene $(2.73 \mathrm{~g}, 2.56 \mathrm{mmol})$ in $60 \mathrm{~mL} \mathrm{CHCl}_{3}$, silver trifluoroacetate $(3.89 \mathrm{~g}, 17.6 \mathrm{mmol})$ and iodine $(4.48 \mathrm{~g}, 17.7 \mathrm{mmol})$. The pure product was separated by silica-gel column chromatography using petroleum ether/ $\mathrm{CH}_{2} \mathrm{Cl}_{2}(\mathrm{v} / \mathrm{v} 8: 1)$ as eluent affording a viscous liquid, $81 \%$ yield $(3.42 \mathrm{~g}) .{ }^{1} \mathrm{H}$ NMR $\left(270 \mathrm{MHz}, \mathrm{CDCl}_{3}, \delta\right) 6.98(\mathrm{~m}, 8 \mathrm{H})$, $4.27(\mathrm{~d}, J=13.23 \mathrm{~Hz}, 4 \mathrm{H}), 3.83(\mathrm{t}, J=7.29 \mathrm{~Hz}, 8 \mathrm{H}), 3.03(\mathrm{~d}, J=13.50 \mathrm{~Hz}, 4 \mathrm{H}), 1.84(\mathrm{~m}, 8 \mathrm{H})$, $1.35(\mathrm{bs}, 48 \mathrm{H}), 0.87(\mathrm{t}, J=8.10 \mathrm{~Hz}, 12 \mathrm{H}) .{ }^{13} \mathrm{C} \mathrm{NMR}\left(67.8 \mathrm{MHz}, \mathrm{CDCl}_{3}, \delta\right) 156.1,136.8,136.6$, 86.0, 31.9, 30.3, 30.1, 29.9, 29.4, 26.2. 22.7, 14.0. MS (FAB) $\mathrm{m} / \mathrm{z} 1434.4\left(\mathrm{M}^{+}\right)$.

5,11,17,23-Tetrakisiodo-25,26,27,28-tetraundecyloxycalix[4]arene 3d. The alkylation procedure was followed using calix[4] arene $(0.99 \mathrm{~g}, 2.33 \mathrm{mmol})$ and 1-bromoundecane $(3.29 \mathrm{~mL}$, $14.00 \mathrm{mmol}$ ). The crude product was filtered by short silica-gel column chromatography using petroleum ether/ $\mathrm{CH}_{2} \mathrm{Cl}_{2}$ (v/v 8:1) as eluent affording (25,26,27,28-tetraundecyloxycalix[4]arene) as a viscous liquid. Following the above iodination procedure using 25,26,27,28tetraundecyloxycalix[4]arene $(1.05 \mathrm{~g}, 1.01 \mathrm{mmol})$ in $70 \mathrm{~mL} \mathrm{CHCl}_{3}$, silver trifluoroacetate $(1.36 \mathrm{~g}$, $6.16 \mathrm{mmol})$ and iodine $(1.54 \mathrm{~g}, 6.06 \mathrm{mmol})$. The pure product was separated by silica-gel column chromatography using petroleum ether $/ \mathrm{CH}_{2} \mathrm{Cl}_{2}(\mathrm{v} / \mathrm{v}$ 8:1) as eluent affording a viscous liquid, $95 \%$ yield (1.48 g). ${ }^{1} \mathrm{H}$ NMR $\left(270 \mathrm{MHz}, \mathrm{CDCl}_{3}, \delta\right) 6.97(\mathrm{~m}, 8 \mathrm{H}), 4.26(\mathrm{~d}, J=13.50 \mathrm{~Hz}$, 4H), $3.78(\mathrm{t}, 8 \mathrm{H}), 3.03(\mathrm{~d}, J=13.23 \mathrm{~Hz}, 4 \mathrm{H}), 1.83(\mathrm{~m}, 8 \mathrm{H}), 1.25(\mathrm{bs}, 64 \mathrm{H}), 0.86(\mathrm{t}, J=8.10 \mathrm{~Hz}$, 12H). ${ }^{13} \mathrm{C} \mathrm{NMR}\left(67.8 \mathrm{MHz}, \mathrm{CDCl}_{3}, \delta\right)$ 156.1, 136.9, 136.6, 86.0, 75.4, 32.0, 30.3, 30.1, 29.9, 29.8, 29.7 29.4, 26.2. 22.7, 14.0. $\mathrm{MS}(\mathrm{FAB}) \mathrm{m} / \mathrm{z} 1544.7\left(\mathrm{M}^{+}\right)$.

5,11,17,23-Tetrakisiodo-25,26,27,28-tetradodecyloxycalix[4]arene 3e. The alkylation procedure was followed using calix[4]arene $(0.51 \mathrm{~g}, 1.19 \mathrm{mmol})$ and 1-bromododecane $(1.80 \mathrm{~mL}$, 
$7.10 \mathrm{mmol})$. The crude product was filtered by short silica-gel column chromatography using petroleum ether/ $\mathrm{CH}_{2} \mathrm{Cl}_{2}$ (v/v 8:1) as eluent affording (25,26,27,28-tetradodecyloxycalix[4]arene) as a viscous liquid. Following the above iodination procedure using 25,26,27,28-tetradodecyloxycalix[4]arene $(0.53 \mathrm{~g}, 0.49 \mathrm{mmol})$ in $15 \mathrm{~mL} \mathrm{CHCl}_{3}$, silver trifluoroacetate $(0.67 \mathrm{~g}, 3.03$ $\mathrm{mmol})$ and iodine $(0.84 \mathrm{~g}, 3.31 \mathrm{mmol})$. The pure product was separated by silica-gel column chromatography using petroleum ether $/ \mathrm{CH}_{2} \mathrm{Cl}_{2}$ (v/v 8:1) as eluent affording a viscous liquid, 80 \% yield (0.62 g). ${ }^{1} \mathrm{H} \mathrm{NMR}\left(270 \mathrm{MHz}, \mathrm{CDCl}_{3}, \delta\right) 6.98(\mathrm{~m}, 8 \mathrm{H}), 4.27(\mathrm{~d}, J=13.50 \mathrm{~Hz}, 4 \mathrm{H}), 3.82$ (t, $J=8.10 \mathrm{~Hz}, 8 \mathrm{H}), 3.04(\mathrm{~d}, J=13.5 \mathrm{~Hz}, 4 \mathrm{H}), 1.84(\mathrm{~m}, 8 \mathrm{H}), 1.26(\mathrm{bs}, 72 \mathrm{H}), 0.86(\mathrm{t}, J=8.10 \mathrm{~Hz}$, 12H). ${ }^{13} \mathrm{C} \mathrm{NMR}\left(67.8 \mathrm{MHz}, \mathrm{CDCl}_{3}\right.$, $\left.\delta\right)$ 156.1, 136.9, 136.6, 86.0, 75.4, 31.9, 30.1, 30.0, 29.9, 29.8, 29.7, 29.6, 29.5, 22.7, 14.0. $\mathrm{MS}(\mathrm{FAB}) \mathrm{m} / \mathrm{z} 1600.4\left(\mathrm{M}^{+}\right)$.

5,11,17,23-Tetrakisiodo-25,26,27,28-tetratridecyloxycalix[4]arene $\quad 3 f . \quad$ The alkylation procedures were followed using 25,26,27,28-tetra-hydroxycalix[4]arene (0.52 g, $1.22 \mathrm{mmol})$ and 1-bromotridecane $(1.93 \mathrm{~mL}, 7.73 \mathrm{mmol})$. The crude product was filtered by short silica-gel column chromatography using petroleum ether $/ \mathrm{CH}_{2} \mathrm{Cl}_{2}$ (v/v 8:1) as eluent affording $(25,26,27,28$-tetratridecyloxycalix[4]arene) as a viscous liquid. Following the above iodination procedure using 25,26,27,28-tetratridodecyloxycalix[4]arene $(0.24 \mathrm{~g}, 0.21 \mathrm{mmol})$ in $20 \mathrm{~mL}$ $\mathrm{CHCl}_{3}$, silver trifluoroacetate $(0.29 \mathrm{~g}, 1.31 \mathrm{mmol})$ and iodine $(0.37 \mathrm{~g}, 1.46 \mathrm{mmol})$. The pure product was separated by silica-gel column chromatography using petroleum ether/ $\mathrm{CH}_{2} \mathrm{Cl}_{2}(\mathrm{v} / \mathrm{v}$ 8:1) as eluent affording a viscous liquid with $92 \%$ yield $(0.32 \mathrm{~g}) .{ }^{1} \mathrm{H} \mathrm{NMR}\left(270 \mathrm{MHz}, \mathrm{CDCl}_{3}, \delta\right)$ $6.98(\mathrm{~m}, 8 \mathrm{H}), 4.27(\mathrm{~d}, J=13.23 \mathrm{~Hz}, 4 \mathrm{H}), 3.82(\mathrm{t}, J=7.56 \mathrm{~Hz}, 8 \mathrm{H}), 3.04(\mathrm{~d}, J=13.23 \mathrm{~Hz}, 4 \mathrm{H})$, $1.84(\mathrm{~m}, 8 \mathrm{H}), 1.29(\mathrm{bs}, 80 \mathrm{H}), 0.87(\mathrm{t}, J=7.02 \mathrm{~Hz}, 12 \mathrm{H}) .{ }^{13} \mathrm{C} \mathrm{NMR}\left(67.8 \mathrm{MHz}, \mathrm{CDCl}_{3}, \delta\right) 156.0$ $136.8,136.5,86.1,75.4,31.9,30.3,30.1,29.9,29.8,29.7,29.6,29.4,26.3,22.7,14.2$. MS (FAB) $m / z 1657.1\left(\mathrm{M}^{+}\right)$. 
5,11,17,23-Tetrakisiodo-25,26,27,28-tetratetradecyloxycalix[4]arene 3g. The alkylation procedure was followed using calix[4]arene $(0.52 \mathrm{~g}, 1.22 \mathrm{mmol})$ and 1-bromotetradecane $(2.03$ $\mathrm{mL}, 7.32 \mathrm{mmol})$. The crude product was filtered by short silica-gel column chromatography using petroleum ether $/ \mathrm{CH}_{2} \mathrm{Cl}_{2} \quad(\mathrm{v} / \mathrm{v} \quad 8: 1)$ as eluent affording $(25,26,27,28$ tetratetradecyloxycalix[4]arene) as a viscous liquid. Following the above iodination procedure using 25,26,27,28-tetratetra- decyloxycalix[4]arene $(1.47 \mathrm{~g}, 1.22 \mathrm{mmol})$ in $70 \mathrm{~mL} \mathrm{CHCl}_{3}$, silver trifluoroacetate $(1.62 \mathrm{~g}, 7.33 \mathrm{mmol})$ and iodine $(1.86 \mathrm{~g}, 7.32 \mathrm{mmol})$. The pure product was separated by silica-gel column chromatography using petroleum ether/ $\mathrm{CH}_{2} \mathrm{Cl}_{2}$ (v/v 8:1) as eluent affording a viscous liquid, $78 \%$ yield $(1.39 \mathrm{~g}) .{ }^{1} \mathrm{H} \mathrm{NMR}\left(270 \mathrm{MHz}, \mathrm{CDCl}_{3}, \delta\right) 6.98(\mathrm{~m}, 8 \mathrm{H})$, $4.26(\mathrm{~d}, J=13.23 \mathrm{~Hz}, 4 \mathrm{H}), 3.81(\mathrm{t}, J=7.02 \mathrm{~Hz}, 8 \mathrm{H}), 3.03(\mathrm{~d}, J=13.50 \mathrm{~Hz}, 4 \mathrm{H}), 1.83(\mathrm{~m}, 8 \mathrm{H})$, $1.25(\mathrm{bs}, 88 \mathrm{H}), 0.86(\mathrm{t}, J=7.02 \mathrm{~Hz}, 12 \mathrm{H}) .{ }^{13} \mathrm{C} \mathrm{NMR}\left(67.8 \mathrm{MHz}, \mathrm{CDCl}_{3_{2}} \delta\right)$ 156.1, 136.8, 136.5, 86.1, 75.4, 31.9, 30.1, 30.0, 29.9, 29.8, 29.7, 29.4, 26.3, 22.7, 14.0. MS (FAB) m/z 1713.2 (M+).

5,11,17,23-Tetrakisiodo-25,26,27,28-tetrapentadecyloxycalix[4]arene $3 \mathrm{~h}$. The alkylation procedure was followed using calix[4]arene $(0.75,1.77 \mathrm{mmol})$ and 1-bromotetrapentadecane (3.09 $\mathrm{mL}, 10.6 \mathrm{mmol})$. The crude product was filtered by short silica-gel column chromatography using petroleum ether $/ \mathrm{CH}_{2} \mathrm{Cl}_{2}$ (v/v 8:1) as eluent affording $(25,26,27,28$ tetratetrapentradecyloxycalix[4]arene) as a viscous liquid. Following the above iodination procedure using 25,26,27,28-tetrapenta- decyloxycalix[4]arene $(0.41 \mathrm{~g}, 0.32 \mathrm{mmol})$ in $30 \mathrm{~mL}$ $\mathrm{CHCl}_{3}$, silver trifluoroacetate $(0.47 \mathrm{~g}, 1.94 \mathrm{mmol})$ and iodine $(0.37 \mathrm{~g}, 1.93 \mathrm{mmol})$. The pure product was separated by silica-gel column chromatography using petroleum ether/ $\mathrm{CH}_{2} \mathrm{Cl}_{2}(\mathrm{v} / \mathrm{v}$ $8: 1)$ as eluent affording a viscous liquid, $95 \%$ yield $(0.57 \mathrm{~g}) .{ }^{1} \mathrm{H}$ NMR $\left(270 \mathrm{MHz}, \mathrm{CDCl}_{3}, \delta\right)$ $6.98(\mathrm{~m}, 8 \mathrm{H}), 4.26(\mathrm{~d}, 4 \mathrm{H}, J=13.50 \mathrm{~Hz}), 3.82(\mathrm{t}, 8 \mathrm{H}), 3.03(\mathrm{~d}, 4 \mathrm{H}, J=13.50 \mathrm{~Hz}), 1.83(\mathrm{~m}, 8$ $\mathrm{H}), 1.25(\mathrm{bs}, 80 \mathrm{H}), 0.87(\mathrm{t}, 12 \mathrm{H}) .{ }^{13} \mathrm{C} \mathrm{NMR}\left(67.8 \mathrm{MHz}, \mathrm{CDCl}_{3}, \delta\right)$ 156.2, 136.9, 136.7, 86.1, 
75.5, 32.0, 30.2, 30.0, 29.9, 29.8, 29.5, 26.3 22.8, 14.2. MS (FAB) $m / z 1773.4\left(\mathrm{M}^{+}\right)$.

5,11,17,23-Tetrakisiodo-25,26,27,28-tetrahexadecyloxycalix[4]arene $3 \mathbf{i} . \quad$ The alkylation procedure was followed using calix[4]arene (1.06 g, $2.50 \mathrm{mmol})$ and 1-bromohexadecane (4.57 $\mathrm{mL}, 15.00 \mathrm{mmol})$. The crude product was filtered by short silica-gel column chromatography using petroleum ether $/ \mathrm{CH}_{2} \mathrm{Cl}_{2} \quad(\mathrm{v} / \mathrm{v} \quad 8: 1)$ as eluent affording $(25,26,27,28$ tetradodecyloxycalix[4]arene) as a viscous liquid. Following the above iodination procedure using 25,26,27,28-tetra- hexadecyloxycalix[4]arene (1.52 g, $1.15 \mathrm{mmol})$ in $70 \mathrm{~mL} \mathrm{CHCl}_{3}$, silver trifluoroacetate $(1.52 \mathrm{~g}, 7.00 \mathrm{mmol})$ and iodine $(1.75 \mathrm{~g}, 6.89 \mathrm{mmol})$. The pure product was separated by silica-gel column chromatography using petroleum ether $/ \mathrm{CH}_{2} \mathrm{Cl}_{2}$ (v/v 8:1) as eluent affording a viscous liquid, $93 \%$ yield (1.95 g). ${ }^{1} \mathrm{H}$ NMR $\left(270 \mathrm{MHz}, \mathrm{CDCl}_{3}, \delta\right) 6.97(\mathrm{~m}, 8 \mathrm{H})$, $4.26(\mathrm{~d}, J=13.50 \mathrm{~Hz}, 4 \mathrm{H}), 3.80(\mathrm{t}, J=7.29 \mathrm{~Hz}, 8 \mathrm{H}), 3.03(\mathrm{~d}, J=13.23 \mathrm{~Hz}, 4 \mathrm{H}), 1.82(\mathrm{~m}, 8 \mathrm{H})$, $1.24(\mathrm{bs}, 104 \mathrm{H}), 0.87(\mathrm{t}, J=7.02 \mathrm{~Hz}, 12 \mathrm{H}) .{ }^{13} \mathrm{C} \mathrm{NMR}\left(67.8 \mathrm{MHz}, \mathrm{CDCl}_{3}, \delta\right) 156.2,136.9,136.7$, 86.1, 75.5, 32.0, 30.4, 30.2, 30.0, 29.9, 29.8, 29.4, 26.3, 22.7, 14.2. MS (FAB) $\mathrm{m} / \mathrm{z} 1825.5\left(\mathrm{M}^{+}\right)$.

\section{5,11,17,23-Tetrakis(4'-methylbiphenyl)-25,26,27,28-tetraoctyloxycalix[4]arene $\quad C_{8}$-Calix-}

OPP(2)-Me. The coupling procedure was followed using 5,11,17,23-tetrakisiodo-25,26,27,28tetraoctyloxycalix[4]arene (433 mg, $0.34 \mathrm{mmol}$ ), 4-bromo-4'-methylbiphenylboronic acid (398 $\mathrm{mg}, 1.88 \mathrm{mmol})$ and $\mathrm{Pd}(\mathrm{OAc})_{2}(42 \mathrm{mg}, 0.19 \mathrm{mmol})$ and $\mathrm{P}(\mathrm{o}-\mathrm{tol})_{3}(112 \mathrm{mg}, 0.37 \mathrm{mmol})$. The crude product was purified by silica-gel column chromatography using petroleum ether as eluent. The pure product was obtained by re-crystallization from petroleum ether affording a white solid of $0.25 \mathrm{~g}(50 \%$ yield $) .{ }^{1} \mathrm{H} \mathrm{NMR}\left(270 \mathrm{MHz}, \mathrm{CDCl}_{3}, \delta\right) 7.34-6.99(\mathrm{~m}, 40 \mathrm{H}), 4.58(\mathrm{~d}, J=12.69$ Hz, 4H), 3.99 (t, 8H), $3.31(\mathrm{~d}, J=13.23 \mathrm{~Hz}, 4 \mathrm{H}), 2.34(\mathrm{~s}, 12 \mathrm{H}), 2.02(\mathrm{~m}, 8 \mathrm{H}), 1.41-1.31$ (bs, 40H), $0.89(\mathrm{t}, J=6.75 \mathrm{~Hz}, 12 \mathrm{H}) .{ }^{13} \mathrm{C} \mathrm{NMR}\left(100 \mathrm{MHz}, \mathrm{CDCl}_{3}, \delta\right) 156.1,139.7,138.7,137.7$, $136.3,135.0,134.7,129.2,127.0,126.8,126.7,126.6,75.6,32.1,31.5,30.1,29.8,26.5,22.8$, 
21.1, 14.2. HRMS (MALDI-TOF) $\mathrm{m} / \mathrm{z}$ calcd for $\mathrm{C}_{112} \mathrm{H}_{128} \mathrm{O}_{4} \mathrm{Na}, 1559.9710$. Found: 1560.9701 $[\mathrm{M}+\mathrm{Na}]^{+}$

\section{5,11,17,23-Tetrakis(4'-methylbiphenyl)-25,26,27,28-tetraundecyloxycalix[4]arene}

Calix-OPP(2)-Me. The coupling procedure was followed using 5,11,17,23-tetrakisiodo25,26,27,28-tetraundecyloxycalix[4]arene $(0.47 \mathrm{~g}, 0.31 \mathrm{mmol})$, 4-bromo-4'-methylbiphenylboronic acid (393 $\mathrm{mg}, 1.85 \mathrm{mmol})$ and $\mathrm{Pd}(\mathrm{OAc})_{2}(41 \mathrm{mg}, 0.18 \mathrm{mmol})$ and $\mathrm{P}(\mathrm{o} \text {-tol })_{3}(56 \mathrm{mg}, 0.18$ mmol). The crude product was purified by silica-gel column chromatography using petroleum ether as eluent. The pure product was obtained by re-crystallization from petroleum ether affording a white solid of $0.35 \mathrm{~g}$ (66 \% yield). ${ }^{1} \mathrm{H} \mathrm{NMR}\left(270 \mathrm{MHz}, \mathrm{CDCl}_{3}, \delta\right)$ 7.34-6.99 (m, 40 H), $4.56(\mathrm{~d}, J=13.50 \mathrm{~Hz}, 4 \mathrm{H}), 3.99(\mathrm{t}, 8 \mathrm{H}), 3.31(\mathrm{~d}, J=13.50 \mathrm{~Hz}, 4 \mathrm{H}), 2.34(\mathrm{~s}, 12 \mathrm{H}), 2.02(\mathrm{~m}$, 8H), 1.42-1.28 (bs, 64H), $0.87(\mathrm{t}, J=7.20 \mathrm{~Hz}, 12 \mathrm{H}) .{ }^{13} \mathrm{C} \mathrm{NMR}\left(100 \mathrm{MHz}, \mathrm{CDCl}_{3}, \delta\right) 156.2$, $139.8,138.7,137.8,136.4,135.1,134.7,129.3,127.0,126.9,126.8,126.6,75.5,32.0,31.4,30.1$, 30.4, 30.1, 29.9, 29.8, 29.5, 26.4, 22.7, 21.1, 14.1. HRMS (MALDI-TOF) $\mathrm{m} / \mathrm{z}$ calcd for $\mathrm{C}_{124} \mathrm{H}_{152} \mathrm{O}_{4} \mathrm{Na}$, 1729.1622. Found 1728.1476 [M+Na] $]^{+}$.

4-cyano-4'-nonyloxybiphenyl $\mathbf{C}_{9}$-OPP(2)-CN. The alkylation procedures were followed using 4-iodophenol (0.98 g, $4.45 \mathrm{mmol})$ and 1-bromononane $(1.1 \mathrm{~mL}, 5.3 \mathrm{mmol})$. The crude product was filtered by short silica-gel column chromatography using petroleum ether/ $\mathrm{CH}_{2} \mathrm{Cl}_{2}(\mathrm{v} / \mathrm{v} 8: 1)$ as eluent affording 4-iodononyloxybenzene as a viscous liquid. The coupling procedure was followed using 4-iodononyloxybenzene $(0.45 \mathrm{~g}, 1.30 \mathrm{mmol})$, 4-cyanobenzeneboronic acid (0.38 $\mathrm{g}, 2.59 \mathrm{mmol})$ and $\mathrm{Pd}(\mathrm{OAc})_{2}(43 \mathrm{mg}, 0.20 \mathrm{mmol})$ and $\mathrm{P}(\mathrm{o}-\mathrm{tol})_{3}(0.12 \mathrm{~g}, 0.39 \mathrm{mmol})$. The crude product was purified by silica-gel column chromatography using $\mathrm{PE} / \mathrm{CH}_{2} \mathrm{Cl}_{2}$ (v/v 10:1) as eluent affording a white solid with an isolated yield of $80 \%(0.33 \mathrm{~g}) .{ }^{1} \mathrm{H} \mathrm{NMR}\left(400 \mathrm{MHz}, \mathrm{CDCl}_{3}, \delta\right)$ 7.68-7.61 (m, 4H), $7.51(\mathrm{~d}, J=8.4 \mathrm{~Hz}, 2 \mathrm{H}), 6.97(\mathrm{~d}, J=8.4 \mathrm{~Hz}, 2 \mathrm{H}), 3.98(\mathrm{t}, J=6.40 \mathrm{~Hz}, 2 \mathrm{H})$, 
1.81-1.77 (m, 2H,), 1.47-1.27 (bs, $12 \mathrm{H}), 0.87(\mathrm{t}, J=7.2 \mathrm{~Hz}, 3 \mathrm{H}) .{ }^{13} \mathrm{C} \mathrm{NMR}(100 \mathrm{MHz}$, $\left.\mathrm{CDCl}_{3}, \delta\right) 159.7,145.2,132.5,131.1,128.3,127.0,119.1,115.0,109.9,68.1,31.8,29.5,29.4$ 29.2, 29.1, 26.0, 22.6, 14.0. HRMS (MALDI-TOF) $\mathrm{m} / \mathrm{z}$ calcd for $\mathrm{C}_{22} \mathrm{H}_{27} \mathrm{ON}, 321.2092$. Found $321.2088[\mathrm{M}]^{+}$.

4-cyano-4'-decyloxybiphenyl $\mathbf{C}_{10}-\mathbf{O P P}(2)-\mathbf{C N}$. The alkylation procedures were followed using 4-iodophenol (1.0 g, $4.54 \mathrm{mmol})$ and 1-bromononane $(1.2 \mathrm{~mL}, 5.4 \mathrm{mmol})$. The crude product was filtered by short silica-gel column chromatography using petroleum ether/ $\mathrm{CH}_{2} \mathrm{Cl}_{2}(\mathrm{v} / \mathrm{v} 8: 1)$ as eluent affording 4-iododecyloxybenzene as a viscous liquid. The coupling procedure was followed using 4-iododecyloxybenzene (0.79 g, $2.20 \mathrm{mmol})$, 4-cyanobenzeneboronic acid (0.65 $\mathrm{g}, 4.38 \mathrm{mmol})$ and $\mathrm{Pd}(\mathrm{OAc})_{2}(73 \mathrm{mg}, 0.33 \mathrm{mmol})$ and $\mathrm{P}(\mathrm{o}-\mathrm{tol})_{3}(0.2 \mathrm{~g}, 0.66 \mathrm{mmol})$. The crude product was purified by silica-gel column chromatography using $\mathrm{PE} / \mathrm{CH}_{2} \mathrm{Cl}_{2}(\mathrm{v} / \mathrm{v} 10: 1)$ as eluent affording a white solid with an isolated yield of $77 \%(0.57 \mathrm{~g}) .{ }^{1} \mathrm{H} \mathrm{NMR}\left(400 \mathrm{MHz}, \mathrm{CDCl}_{3}, \delta\right)$ 7.66-7.60 (m, 4H), $7.50(\mathrm{~d}, J=8.4 \mathrm{~Hz}, 2 \mathrm{H}), 6.97(\mathrm{~d}, J=8.4 \mathrm{~Hz}, 2 \mathrm{H}), 3.98(\mathrm{t}, J=6.40 \mathrm{~Hz}, 2 \mathrm{H})$, 1.81-1.77 (m, 2H), 1.47-1.27 (bs, $14 \mathrm{H}), 0.88(\mathrm{t}, J=6.80 \mathrm{~Hz}, 3 \mathrm{H}) .{ }^{13} \mathrm{C} \mathrm{NMR}(100 \mathrm{MHz}$, $\left.\mathrm{CDCl}_{3}, \delta\right) 159.7,145.1,132.4,131.0,128.2,126.9,119.0,114.9,109.8,68.0,31.8,29.5,29.4$ 29.3, 29.2, 29.1, 26.0, 22.6, 14.0. HRMS (MALDI-TOF) $\mathrm{m} / \mathrm{z}$ calcd for $\mathrm{C}_{23} \mathrm{H}_{29} \mathrm{ON}, 335.2249$. Found $335.2250[\mathrm{M}]^{+}$.

4-cyano-4'-undecyloxybiphenyl $\mathbf{C}_{11}-\mathbf{O P P}(2)-\mathbf{C N}$. The alkylation procedures were followed using 4-iodophenol (0.60 g, $2.73 \mathrm{mmol})$ and 1-bromoundecane $(0.96 \mathrm{~mL}, 4.0 \mathrm{mmol})$. The crude product was filtered by short silica-gel column chromatography using petroleum ether $/ \mathrm{CH}_{2} \mathrm{Cl}_{2}$ (v/v 8:1) as eluent affording 4-iodoundecyloxybenzene as a viscous liquid. The coupling procedure was followed using 4-iodoundecyloxybenzene $(0.41 \mathrm{~g}, 1.10 \mathrm{mmol})$, 4cyanobenzeneboronic acid $(0.32 \mathrm{~g}, 2.2 \mathrm{mmol})$ and $\mathrm{Pd}(\mathrm{OAc})_{2}(37 \mathrm{mg}, 0.16 \mathrm{mmol})$ and $\mathrm{P}(\mathrm{o}-\mathrm{tol})_{3}$ $(0.1 \mathrm{~g}, 0.33 \mathrm{mmol})$. The crude product was purified by silica-gel column chromatography using 
$\mathrm{PE} / \mathrm{CH}_{2} \mathrm{Cl}_{2}(\mathrm{v} / \mathrm{v} 10: 1)$ as eluent affording a white solid with an isolated yield of $85 \%(0.33 \mathrm{~g})$. ${ }^{1} \mathrm{H}$ NMR (270 MHz, $\left.\mathrm{CDCl}_{3}, \delta\right)$ 7.66-7.61 (m, 4H), $7.50(\mathrm{~d}, J=7.83 \mathrm{~Hz}, 2 \mathrm{H}), 6.97(\mathrm{~d}, J=8.1$ $\mathrm{Hz}, 2 \mathrm{H}), 3.98(\mathrm{t}, J=6.48 \mathrm{~Hz}, 2 \mathrm{H}), 1.82-1.74(\mathrm{~m}, 2 \mathrm{H}), 1.46-1.27(\mathrm{bs}, 16 \mathrm{H}), 0.88(\mathrm{t}, J=7.02 \mathrm{~Hz}$, 3H). ${ }^{13} \mathrm{C} \mathrm{NMR}\left(66 \mathrm{MHz}, \mathrm{CDCl}_{3}, \delta\right) 159.5,145.0,132.3,130.9,128.1,126.8,118.9,114.9$, 109.8, 68.0, 31.9, 29.6, 29.4, 29.3, 29.2, 26.0, 22.7, 14.2. HRMS (MALDI-TOF) $\mathrm{m} / \mathrm{z}$ calcd for $\mathrm{C}_{24} \mathrm{H}_{31} \mathrm{ON}, 349.2405$. Found 349.2397 [M] $]^{+}$.

4-cyano-4'-dodecyloxybiphenyl $\mathbf{C}_{12}-\mathbf{O P P}(2)-\mathbf{C N}$. The alkylation procedures were followed using 4-iodophenol (0.61 g, $2.77 \mathrm{mmol})$ and 1-bromododecane (1.1 mL, $4.41 \mathrm{mmol})$. The crude product was filtered by short silica-gel column chromatography using petroleum ether $/ \mathrm{CH}_{2} \mathrm{Cl}_{2}$ (v/v 8:1) as eluent affording 4-iodododecyloxybenzene as a viscous liquid. The coupling procedure was followed using 4-iodododecyloxybenzene $(0.46 \mathrm{~g}, 1.19 \mathrm{mmol})$, 4cyanobenzeneboronic acid $(0.35 \mathrm{~g}, 2.38 \mathrm{mmol})$ and $\mathrm{Pd}(\mathrm{OAc})_{2}(40 \mathrm{mg}, 0.18 \mathrm{mmol})$ and $\mathrm{P}(\mathrm{o} \text {-tol })_{3}$ $(0.1 \mathrm{~g}, 0.36 \mathrm{mmol})$. The crude product was purified by silica-gel column chromatography using $\mathrm{PE} / \mathrm{CH}_{2} \mathrm{Cl}_{2}(\mathrm{v} / \mathrm{v} 10: 1)$ as eluent affording a white solid with an isolated yield of $75 \%(0.32 \mathrm{~g})$. ${ }^{1} \mathrm{H}$ NMR $\left(400 \mathrm{MHz}, \mathrm{CDCl}_{3}, \delta\right)$ 7.68-7.61 (m, 4H), $7.51(\mathrm{~d}, J=8.4 \mathrm{~Hz}, 2 \mathrm{H}), 6.97(\mathrm{~d}, J=8.4 \mathrm{~Hz}$, 2H), $3.98(\mathrm{t}, J=6.0 \mathrm{~Hz}, 2 \mathrm{H}), 1.79(\mathrm{~m}, 2 \mathrm{H}), 1.30-1.25(\mathrm{bs}, 16 \mathrm{H}), 0.86(\mathrm{t}, J=7.20 \mathrm{~Hz}, 3 \mathrm{H}) .{ }^{13} \mathrm{C}$ NMR (100 MHz, $\left.\mathrm{CDCl}_{3}, \delta\right)$ 159.7, 145.2, 132.5, 131.2, 128.3, 127.0, 119.1, 115.0, 110.0, 68.1, 31.9, 29.6, 29.5 , 29.4 , 29.3, 29.2, 26.0, 22.7, 14.1. HRMS (MALDI-TOF) $\mathrm{m} / \mathrm{z}$ calcd for $\mathrm{C}_{25} \mathrm{H}_{33} \mathrm{ON}, 363.2562$. Found 363.2545 [M] $]^{+}$. 


\section{Differential Scanning Calorimetry (DSC) Results}

DSC thermal analysis diagram for $C_{n}-$ Calix-OPP(1)-CN, $n=9-16$

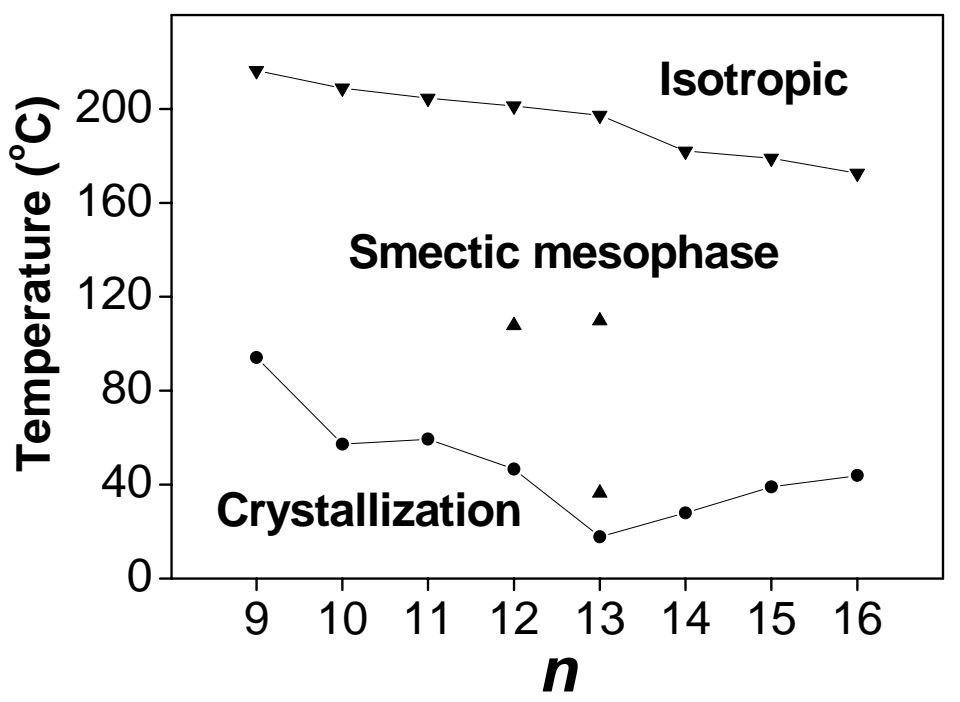

A phase diagram showing the smectic phase range of $\mathrm{C}_{n}$-CalixOPP(1)-CN versus the tail alkoxy carbon number $n=9-16$ measured from the second heating cycle (data in Table 1).

\section{DSC thermal analysis for $\mathrm{C}_{n}-\mathrm{Calix}-\mathrm{OPP}(2)-\mathrm{CH}_{3}, n=9,10$}

In contrary to that reproducible DSC traces were always obtained for $\mathrm{C}_{n}$-Calix-OPP(1)-CN samples, the phase behavior of $\mathrm{C}_{10}$-Calix-OPP(2)- $\mathrm{CH}_{3}$ exhibits two small endothermic transitions with the peak temperatures at 137 and $186{ }^{\circ} \mathrm{C}$ on the second and subsequent heating cycles while no detectable thermal transition on the cooling cycles. $\mathrm{C}_{9}$-Calix-OPP(2)- $\mathrm{CH}_{3}$ shows two endothermic peaks of 128 and $196^{\circ} \mathrm{C}$ on the first heating cycle and only one peak of $196^{\circ} \mathrm{C}$ on the second and subsequent heating cycles and a broad exothermic transition with peak at 147 ${ }^{\circ} \mathrm{C}$ on the cooling cycles. Moreover, for $\mathrm{C}_{9}-\mathrm{Calix}-\mathrm{OPP}(2)-\mathrm{CH}_{3}$, when the temperature was raised much higher than the isotropic temperature $\left(196{ }^{\circ} \mathrm{C}\right)$, such as $220{ }^{\circ} \mathrm{C}$, then no any thermal transition could be detected from either the heating or cooling cycles, even the temperature was lowered to $-40{ }^{\circ} \mathrm{C}$. So for the absence of strong dipole interactions, the self-organization of $\mathrm{C}_{9}$ - 
and $\mathrm{C}_{10}$-Calix-OPP(2)- $\mathrm{CH}_{3}$ is a much slower process compared with that of $\mathrm{C}_{n}$-Calix-OPP(1)$\mathrm{CN}$.

Table S1.1. DSC data for $\mathbf{C}_{n}$-Calix-OPP(2)- $\mathbf{C H}_{3}(n=9,10)$

\begin{tabular}{c|lc|c}
\hline \multirow{2}{*}{ Samples } & \multicolumn{2}{|c|}{ Heating } & Cooling \\
\hline $\mathbf{C}_{\mathbf{9}}$-Calix-OPP(2)-CH $\mathbf{C H}_{3}$ & $128^{\circ} \mathrm{C}(1 \mathrm{st})$ & $196.0^{\circ} \mathrm{C}$ & $147.0^{\circ} \mathrm{C}$ \\
& $(27.5 \mathrm{~J} / \mathrm{g})$ & $(12.5 \mathrm{~J} / \mathrm{g})$ & $(-9.80 \mathrm{~J} / \mathrm{g})$ \\
\hline $\mathbf{C}_{\mathbf{1 0}}$-Calix-OPP(2)-CH & $136.9^{\circ} \mathrm{C}$ & $186.2^{\circ} \mathrm{C}$ & No thermal transition \\
& $(0.73 \mathrm{~J} / \mathrm{g})$ & $(1.98 \mathrm{~J} / \mathrm{g})$ & detected \\
\hline
\end{tabular}
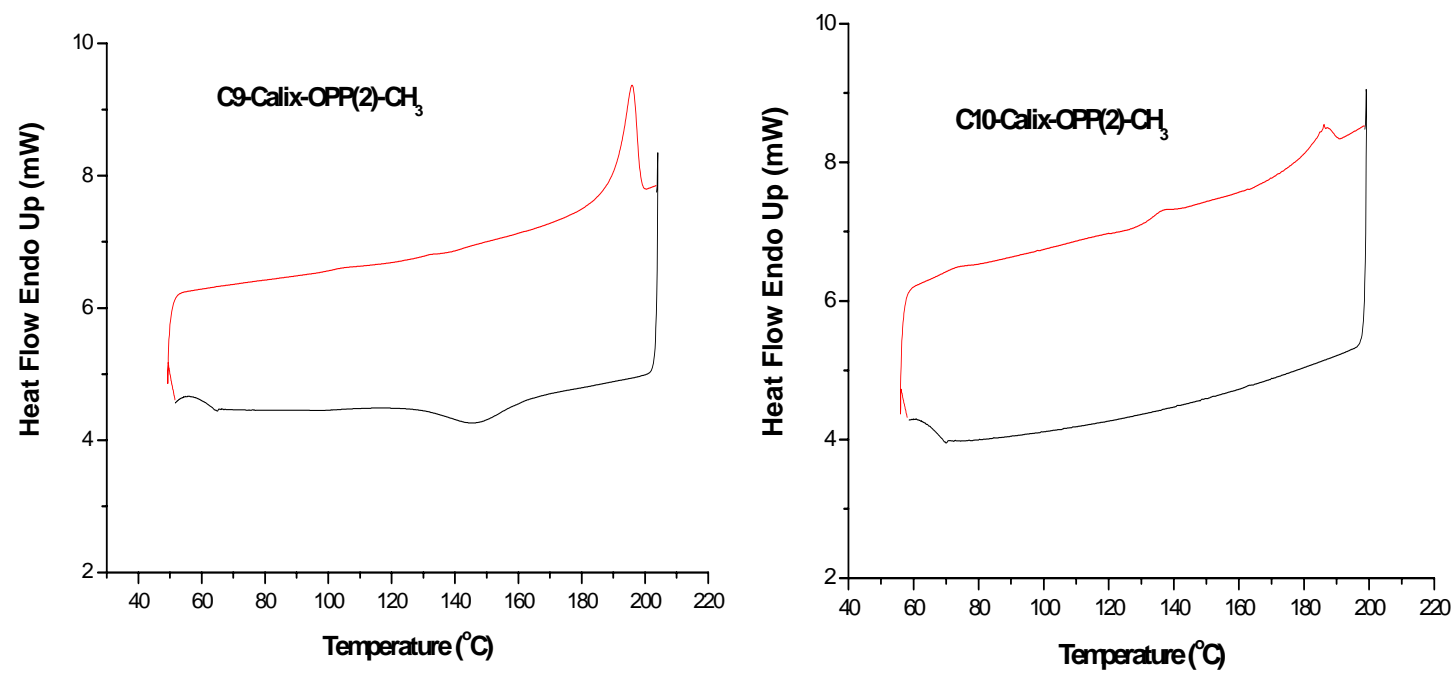

DSC thermal analysis for monomer compounds Cn-OPP(2)-CN, $n=$ 9-12

Gray et al. ${ }^{[1,2]}$ reported the synthesis and thermotropic LC properties of the 4-cyano-4'-nalkoxybiphenyl homologues up to 4-cyano-4'-nonyloxybiphenyl (that is Cn-OPP(2)-CN, n=1-9), the lower members of the homologous series showed monotropic or enantiotropic nematic LC phase, while the higher homologues also exhibited smectic A phase. Our results of $\mathrm{C}_{9}-\mathrm{OPP}(2)-$ $\mathrm{CN}$ agree with Gray's thermal transition temperatures of 4-cyano-4'-nonyloxybiphenyl very well $^{[1]}$. 
Table S1.2.DSC thermal analysis data for monomer $\mathbf{C}_{n}-\mathbf{O P P}(2)-\mathbf{C N}(n=9-12)$

\begin{tabular}{|c|c|c|c|c|c|c|c|c|}
\hline Samples & \multicolumn{4}{|c|}{ Heating } & \multicolumn{4}{|c|}{ Cooling } \\
\hline $\mathrm{C}_{9}$ & K1 58.6 & $\begin{array}{c}\text { K2 } 65.3 \\
(93.1)\end{array}$ & $\begin{array}{c}\mathbf{S}_{\mathbf{A}} 76.0 \\
(0.99)\end{array}$ & $\begin{array}{c}\mathbf{N} 78.3 \mathbf{I} \\
(2.01)\end{array}$ & $\begin{array}{r}\text { K1 } 29.8 \\
(-90.5)\end{array}$ & K2 31.4 & $\begin{array}{c}\mathrm{S}_{\mathrm{A}} 74.7 \\
(-0.70)\end{array}$ & $\begin{array}{c}\mathbf{N} 77.0 \mathbf{I} \\
(-2.16)\end{array}$ \\
\hline $\mathrm{C}_{10}$ & $\begin{array}{c}\text { K1 } 53.8 \\
(14.3)\end{array}$ & $\begin{array}{c}\text { K2 } 60.2 \\
(83.1)\end{array}$ & $\begin{array}{r}\mathbf{S}_{\mathrm{A}} 81.6 \\
(8.32)\end{array}$ & I & $\begin{array}{r}\text { K1 } 30.9 \\
(-83.3)\end{array}$ & K2 33.5 & $\begin{array}{r}\mathrm{S}_{\mathrm{A}} 80.3 \\
(-8.23)\end{array}$ & I \\
\hline $\mathrm{C}_{11}$ & $\begin{array}{c}\text { K1 } 62.9 \\
(11.9)\end{array}$ & $\begin{array}{c}\text { K2 } 72.0 \\
(116.9)\end{array}$ & $\begin{array}{r}\mathrm{S}_{\mathrm{A}} 87.6 \\
(10.4)\end{array}$ & I & $\begin{array}{l}\text { K1 45.5 } \\
(-120.6)\end{array}$ & K2 47.2 & $\begin{array}{r}\mathrm{S}_{\mathrm{A}} 85.4 \\
(-10.4)\end{array}$ & I \\
\hline$C_{12}$ & $\begin{array}{c}\text { K1 } 58.1 \\
(5.51)\end{array}$ & $\begin{array}{c}\text { K2 } 69.8 \\
(106.0)\end{array}$ & $\begin{array}{r}\mathbf{S}_{\mathbf{A}} 89.6 \\
(11.2)\end{array}$ & I & K1 47.1 & $\begin{array}{l}\text { K2 } 47.8 \\
(-104.4)\end{array}$ & $\begin{array}{r}\mathrm{S}_{\mathrm{A}} 88.2 \\
(-11.2)\end{array}$ & I \\
\hline
\end{tabular}

Thermal transition temperature in ${ }^{\circ} \mathrm{C}$ (in parentheses, transition enthalpy in $\mathrm{J} / \mathrm{g}$, positive value stands for endothermic, negative value means exothermic); data from the second heating and first cooling cycles. K1, K2: Crystal form 1 and 2; SA: smectic A; N: nematic; I: isotropic.

[1] Gray, G. W.; Mosley, A. J. Chem. Soc., Perkin II, 1976, 97.

[2] Gray, G. W. J. Physique, 1975, 36, C1(3), 337. 
Some Representative Polarized Optical Microscopy (POM) Textures

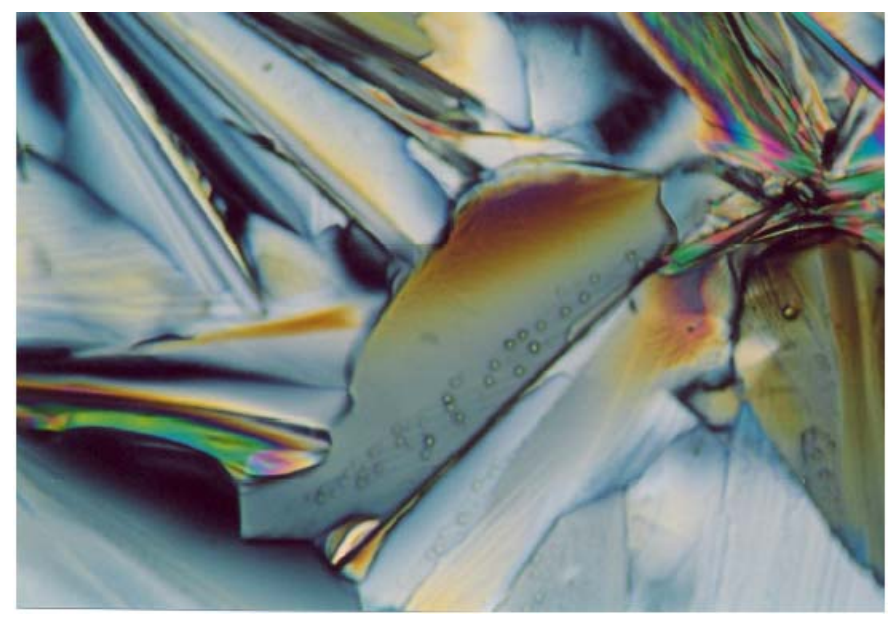

Plate 1. $\mathrm{C}_{9}$-Calix-OPP(1)-CN, cooling to $162^{\circ} \mathrm{C}$, the natural mosaic texture

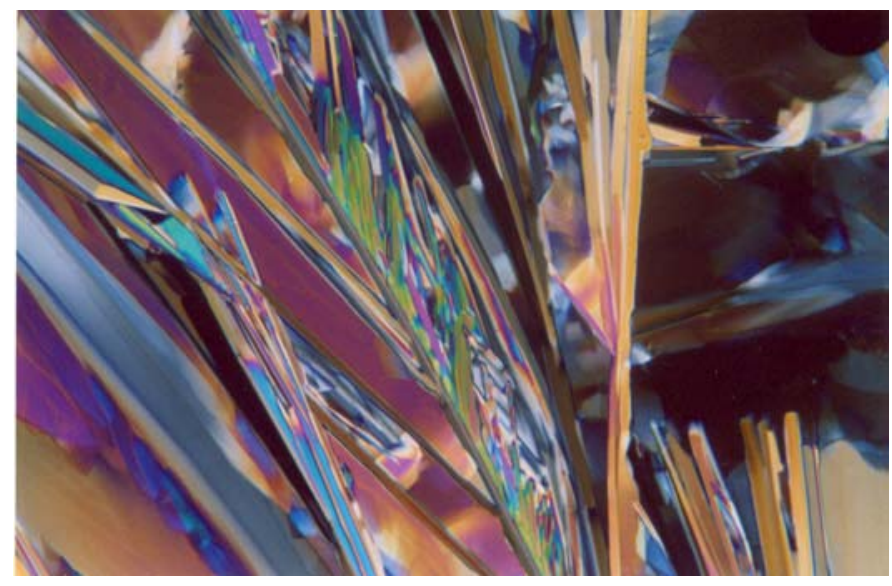

Plate 2. $\mathrm{C}_{10}$-Calix-OPP(1)-CN, $168^{\circ} \mathrm{C}$, mosaic and lancet texture with homeotropic area

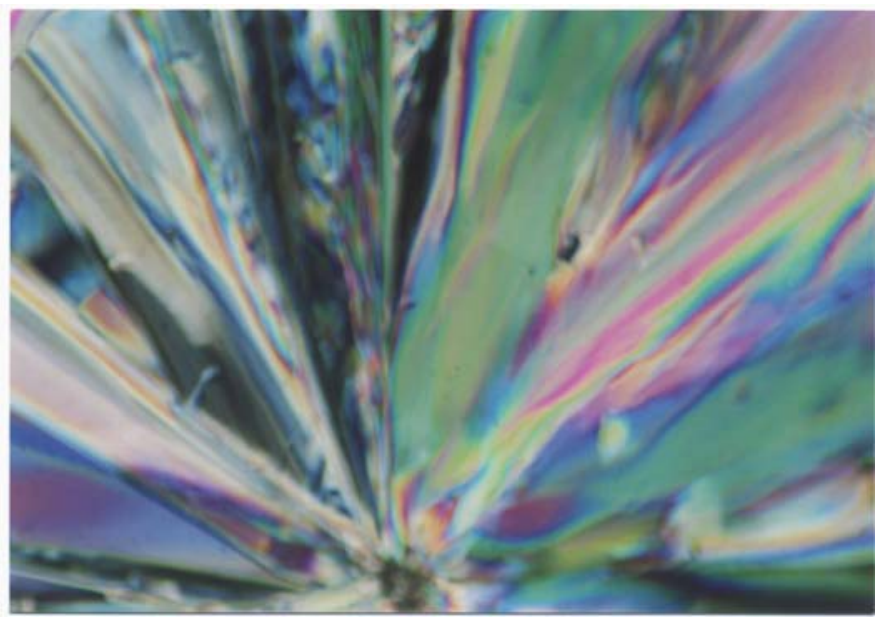

Plate 3. $\mathrm{C}_{12}$-Calix-OPP(1)- $\mathrm{CN}$, cooling to $170^{\circ} \mathrm{C}$, fan-like texture 


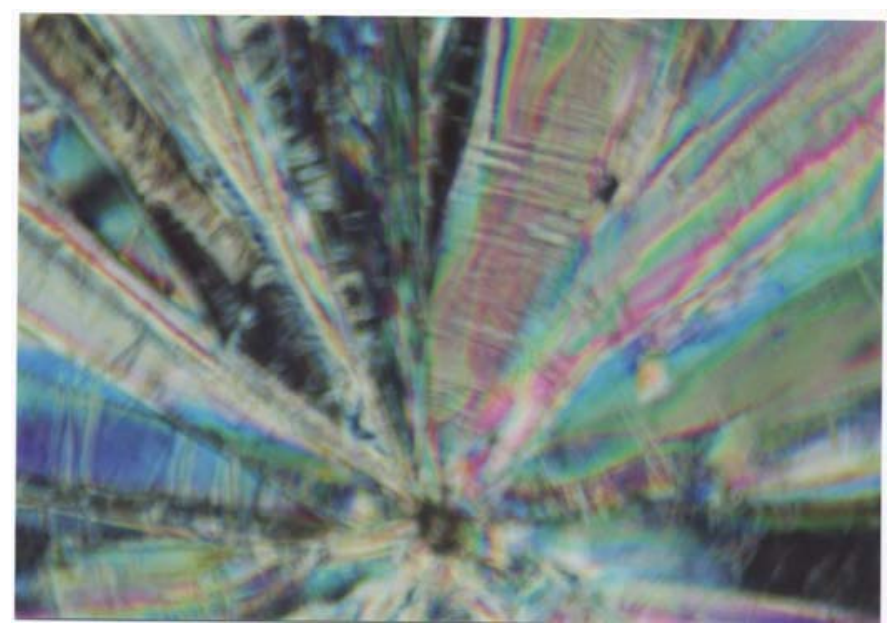

Plate 4. $\mathrm{C}_{12}$-Calix-OPP(1)-CN, cooling to $85^{\circ} \mathrm{C}$, the paramorphotic arced fan-like texture.

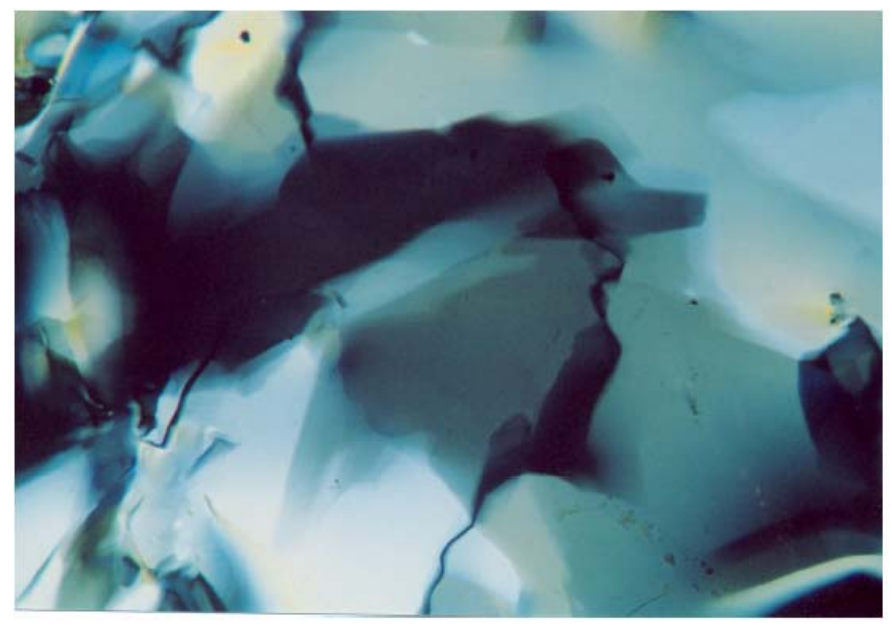

Plate 5. $\mathrm{C}_{13}-\mathrm{Calix}-\mathrm{OPP}(1)-\mathrm{CN}$, cooling to $165^{\circ} \mathrm{C}$, mosaic texture with homeotropic black area

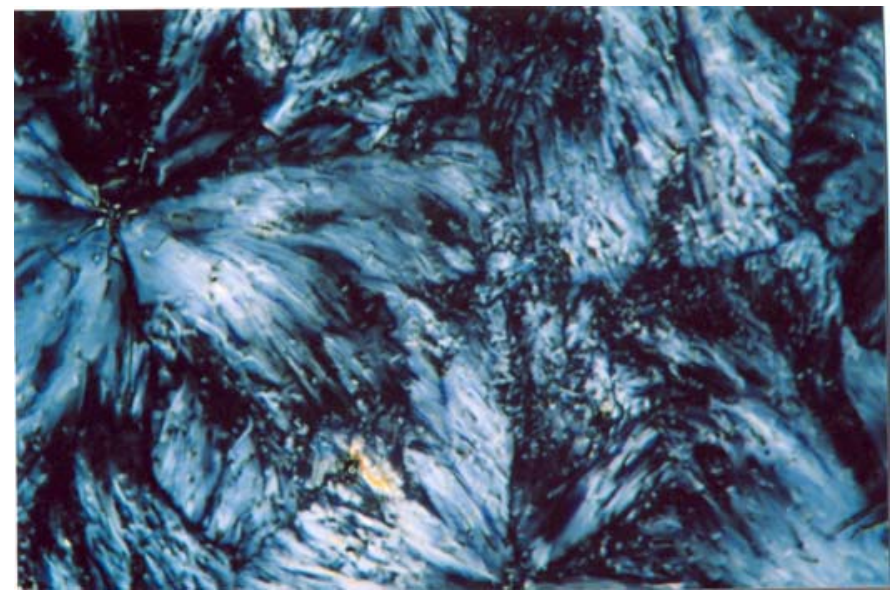

Plate 6. $\mathrm{C}_{16}$-Calix-OPP(1)-CN cooling to $117^{\circ} \mathrm{C}$, fan-like structure formation from grass-like texture 


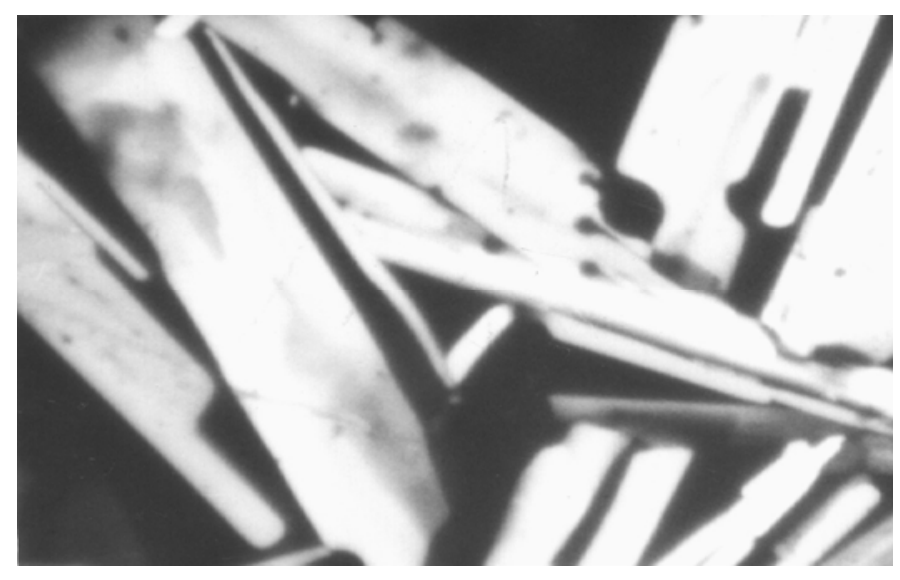

Plate 7. $\mathrm{C}_{10}$-Calix-OPP(2)- $\mathrm{CH}_{3}$ annealed at $160^{\circ} \mathrm{C}$, platelet mosaic texture with homeotropic black area.

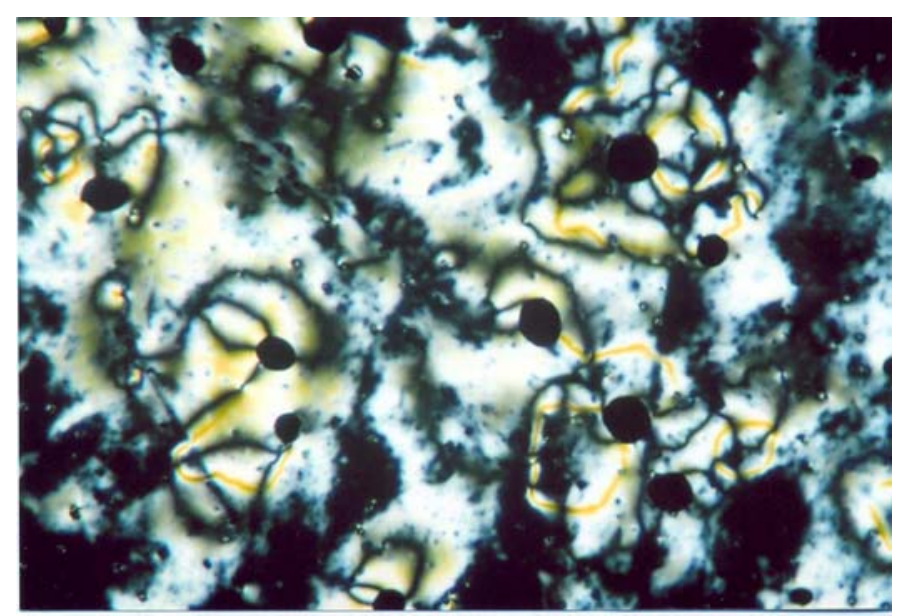

Plate 8. Monomer $\mathrm{C}_{9}-\mathrm{OPP}(2)-\mathrm{CN}$, typical schlieren texture of nematic $\mathrm{LC}$, cooling to $76^{\circ} \mathrm{C}$

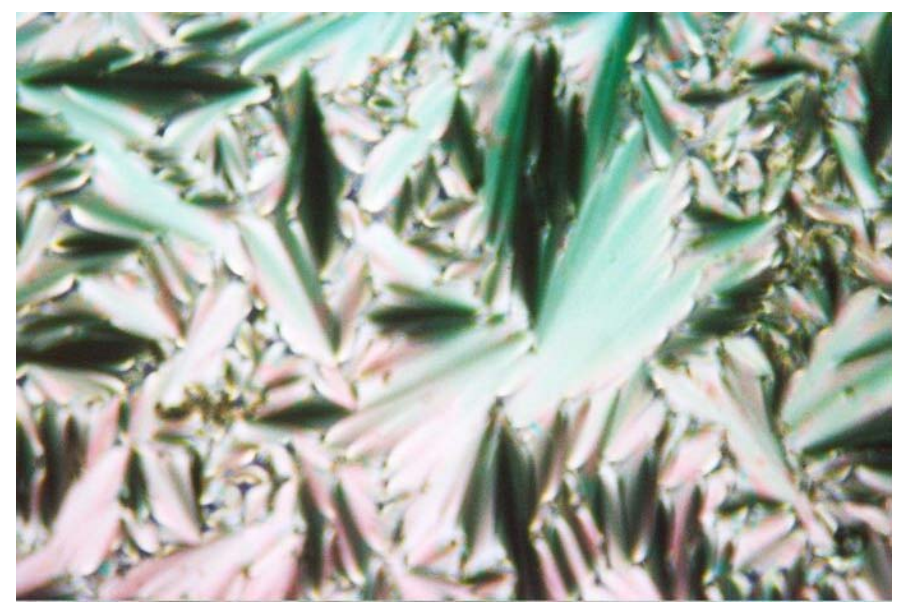

Plate 9. Monomer $\mathrm{C}_{9}-\mathrm{OPP}(2)-\mathrm{CN}$, typical focal conic fan texture of $\mathrm{Sm} \mathrm{A}$, cooling to $72^{\circ} \mathrm{C}$ 


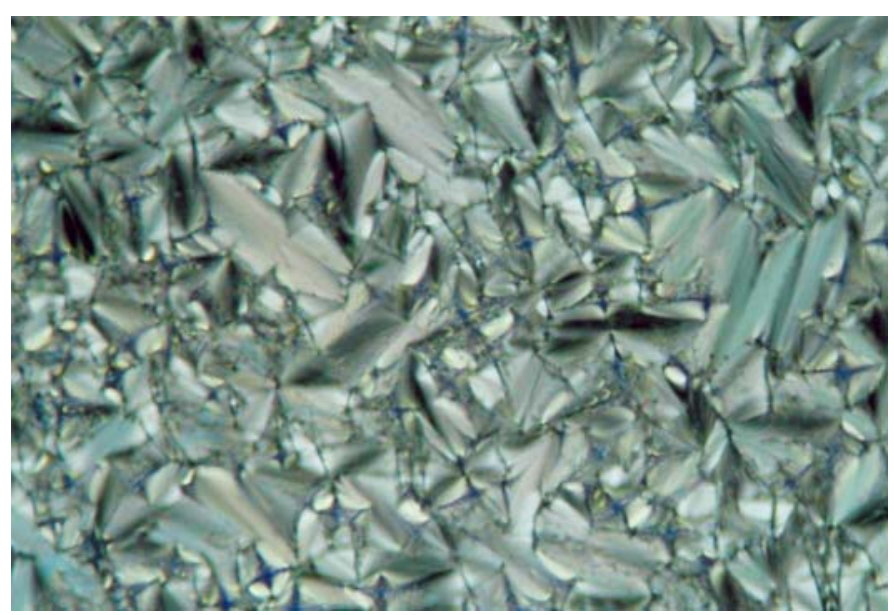

Plate10. Monomer $\mathrm{C}_{10}-\mathrm{OPP}(2)-\mathrm{CN}$, focal conic texture of smectic $\mathrm{A}$, cooling to $64^{\circ} \mathrm{C}$

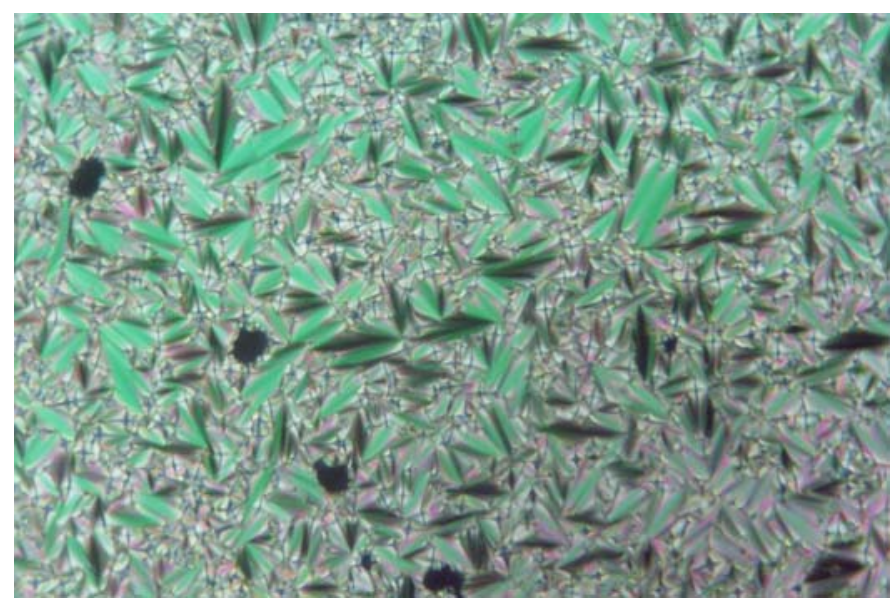

Plate11. Monomer $\mathrm{C}_{11}-\mathrm{OPP}(2)-\mathrm{CN}$, focal conic texture of smectic $\mathrm{A}$, cooling to $78^{\circ} \mathrm{C}$

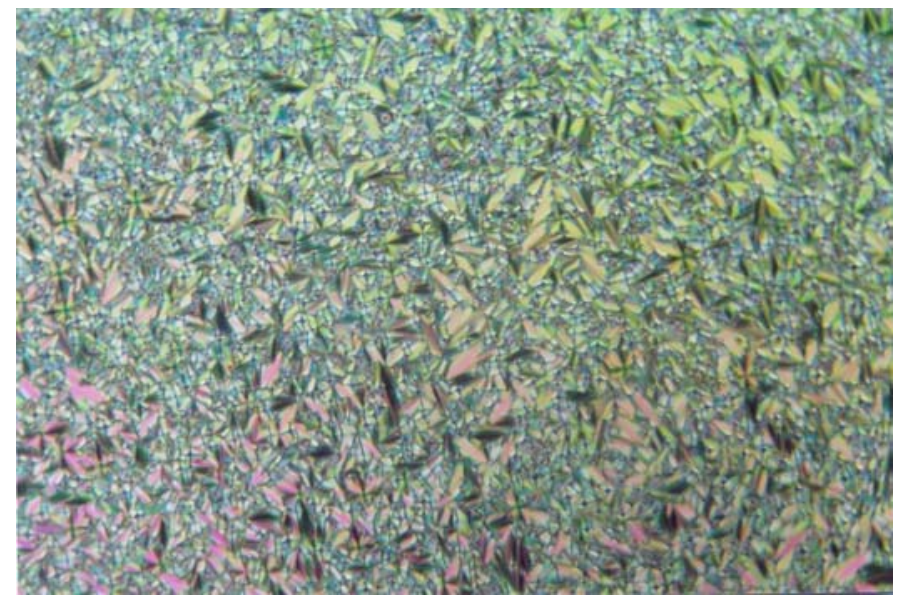

Plate12. Monomer $\mathrm{C}_{12}-\mathrm{OPP}(2)-\mathrm{CN}$, focal conic texture of smectic $\mathrm{A}$, cooling to $80^{\circ} \mathrm{C}$ 


\section{XRD Experimental Results}

The reflection peak positions, the corresponding lattice spacing $d$, relative intensity, and the proposed indexing for the XRD patterns shown in Figure 2 are summarized in Table S2-1 through Table S2-8.

Table S2-1. XRD data for $\mathrm{C}_{9}$-Calix-OPP(1)-CN

\begin{tabular}{|c|c|c|c|}
\hline $\begin{array}{c}\text { peak position } \\
\left(2 \theta /{ }^{\circ}\right)\end{array}$ & $\begin{array}{c}\text { corresponding } d \text { spacing } \\
(\AA) \\
\text { (relative intensity, \%) }\end{array}$ & $\begin{array}{c}\text { relative ratio of } \\
\text { reciprocal } d\end{array}$ & $\begin{array}{c}\text { proposed } \\
\text { indexing }\end{array}$ \\
\hline 3.380 & $26.1(100)$ & 1.00 & $(001)$ \\
\hline 6.740 & $13.1(68.1)$ & 1.99 & $(002)$ \\
\hline 16.84 & $5.26(6.87)$ & 4.96 & $(005)$ \\
\hline 20.26 & $4.38(1.75)$ & 5.96 & $(006)$ \\
\hline
\end{tabular}

Table S2-2. XRD data for $\mathrm{C}_{10}$-Calix-OPP(1)-CN

\begin{tabular}{|c|c|c|c|}
\hline $\begin{array}{c}\text { peak position } \\
\left(2 \theta /{ }^{\circ}\right)\end{array}$ & $\begin{array}{c}\text { corresponding } d \text { spacing } \\
(\AA) \\
(\text { relative intensity, \%) }\end{array}$ & $\begin{array}{c}\text { relative ratio of } \\
\text { reciprocal } d\end{array}$ & $\begin{array}{c}\text { proposed } \\
\text { indexing }\end{array}$ \\
\hline 3.300 & $26.8(100)$ & 1.00 & $(001)$ \\
\hline 6.580 & $13.4(50.3)$ & 2.00 & $(002)$ \\
\hline 9.840 & $8.98(0.51)$ & 2.98 & $(003)$ \\
\hline 13.12 & $6.74(0.92)$ & 3.98 & $(004)$ \\
\hline 16.42 & $5.39(1.72)$ & 4.97 & $(005)$ \\
\hline
\end{tabular}

Table S2-3. XRD data for $\mathrm{C}_{11}$-Calix-OPP(1)-CN

\begin{tabular}{|c|c|c|c|}
\hline $\begin{array}{c}\text { peak position } \\
\left(2 \theta /{ }^{\circ}\right)\end{array}$ & $\begin{array}{c}\text { corresponding } d \text { spacing } \\
(\AA) \\
(\text { relative intensity, \%) }\end{array}$ & $\begin{array}{c}\text { relative ratio of } \\
\text { reciprocal } d\end{array}$ & $\begin{array}{c}\text { proposed } \\
\text { indexing }\end{array}$ \\
\hline 3.180 & $27.8(100)$ & 1.00 & $(001)$ \\
\hline 6.340 & $13.9(63.2)$ & 2.00 & $(002)$ \\
\hline 9.480 & $9.32(2.18)$ & 2.98 & $(003)$ \\
\hline 12.64 & $7.00(0.64)$ & 3.97 & $(004)$ \\
\hline 15.82 & $5.60(3.91)$ & 4.96 & $(005)$ \\
\hline
\end{tabular}


Table S2-4. XRD data for $\mathrm{C}_{12}$-Calix-OPP(1)-CN

\begin{tabular}{|c|c|c|c|}
\hline $\begin{array}{c}\text { peak position } \\
\left(2 \theta /{ }^{\circ}\right)\end{array}$ & $\begin{array}{c}\text { corresponding } d \text { spacing } \\
(\AA) \\
(\text { relative intensity, \%) }\end{array}$ & $\begin{array}{c}\text { relative ratio of } \\
\text { reciprocal } d\end{array}$ & $\begin{array}{c}\text { proposed } \\
\text { indexing }\end{array}$ \\
\hline 2.480 & $35.6(4.08)$ & & \\
\hline 3.040 & $29.0(100)$ & 1.00 & $(001)$ \\
\hline 6.040 & $14.6(45.4)$ & 1.99 & $(002)$ \\
\hline 9.040 & $9.77(4.61)$ & 2.97 & $(003)$ \\
\hline 15.06 & $5.88(3.02)$ & 4.93 & $(005)$ \\
\hline 18.10 & $4.90(0.91)$ & 5.92 & $(006)$ \\
\hline 21.10 & $4.21(0.50)$ & 6.89 & $(007)$ \\
\hline
\end{tabular}

Table S2-5. XRD data for $\mathrm{C}_{13}$-Calix-OPP(1)-CN

\begin{tabular}{|c|c|c|c|}
\hline $\begin{array}{c}\text { peak position } \\
\left(2 \theta /{ }^{\circ}\right)\end{array}$ & $\begin{array}{c}\text { corresponding } d \text { spacing } \\
(\AA) \\
(\text { relative intensity, \%) }\end{array}$ & $\begin{array}{c}\text { relative ratio of } \\
\text { reciprocal } d\end{array}$ & $\begin{array}{c}\text { proposed } \\
\text { indexing }\end{array}$ \\
\hline 2.800 & $31.5(100)$ & 1.00 & $(001)$ \\
\hline 5.560 & $15.9(22.6)$ & 1.98 & $(002)$ \\
\hline 8.320 & $10.6(5.66)$ & 2.97 & $(003)$ \\
\hline 16.64 & $5.32(3.56)$ & 5.92 & $(006)$ \\
\hline 19.44 & $4.56(0.63)$ & 6.91 & $(007)$ \\
\hline 22.26 & $3.99(0.78)$ & 7.89 & $(008)$ \\
\hline
\end{tabular}

Table S2-6. XRD data for $\mathrm{C}_{14}$-Calix-OPP(1)-CN

\begin{tabular}{|c|c|c|c|}
\hline $\begin{array}{c}\text { peak position } \\
\left(2 \theta /{ }^{\circ}\right)\end{array}$ & $\begin{array}{c}\text { corresponding } d \text { spacing } \\
(\AA) \\
\text { (relative intensity, \%) }\end{array}$ & $\begin{array}{c}\text { relative ratio of } \\
\text { reciprocal } d\end{array}$ & $\begin{array}{c}\text { proposed } \\
\text { indexing }\end{array}$ \\
\hline 2.500 & $35.3(16.4)$ & & \\
\hline 2.720 & $32.5(100)$ & 1.00 & $(001)$ \\
\hline 5.380 & $16.4(3.74)$ & 1.98 & $(002)$ \\
\hline 8.120 & $10.9(2.81)$ & 2.98 & $(003)$ \\
\hline 16.12 & $5.49(0.77)$ & 5.92 & $(006)$ \\
\hline
\end{tabular}

Table S2-7. XRD data for $\mathrm{C}_{15}$-Calix-OPP(1)-CN

\begin{tabular}{|c|c|c|c|}
\hline $\begin{array}{c}\text { peak position } \\
\left(2 \theta /{ }^{\circ}\right)\end{array}$ & $\begin{array}{c}\text { corresponding } d \text { spacing } \\
(\AA) \\
(\text { relative intensity, \%) }\end{array}$ & $\begin{array}{c}\text { relative ratio of } \\
\text { reciprocal } d\end{array}$ & $\begin{array}{c}\text { proposed } \\
\text { indexing }\end{array}$ \\
\hline 2.680 & $32.9(100)$ & 1.00 & $(001)$ \\
\hline 7.980 & $11.1(11.4)$ & 2.96 & $(002)$ \\
\hline 15.98 & $5.54(1.43)$ & 5.94 & $(003)$ \\
\hline
\end{tabular}


Table S2-8. XRD data for $\mathrm{C}_{16}$-Calix-OPP(1)-CN

\begin{tabular}{|c|c|c|c|}
\hline $\begin{array}{c}\text { peak position } \\
\left(2 \theta /{ }^{\circ}\right)\end{array}$ & $\begin{array}{c}\text { corresponding } d \text { spacing } \\
(\AA) \\
\text { (relative intensity, \%) }\end{array}$ & $\begin{array}{c}\text { relative ratio of } \\
\text { reciprocal } d\end{array}$ & $\begin{array}{c}\text { proposed } \\
\text { indexing }\end{array}$ \\
\hline 2.380 & $37.1(29.3)$ & & \\
\hline 2.580 & $34.2(100)$ & 1.00 & $(001)$ \\
\hline 7.680 & $11.5(10.3)$ & 2.97 & $(002)$ \\
\hline 15.36 & $5.76(1.54)$ & 5.94 & $(003)$ \\
\hline
\end{tabular}

The lamellar periods $\boldsymbol{d}$ were extracted from XRD data in Table S2-1 through Table S2-8 and plotted as a function of the alkyl carbon number $\boldsymbol{n}$ for samples of $\mathrm{C}_{n}$-Calix-OPP(1)-CN.

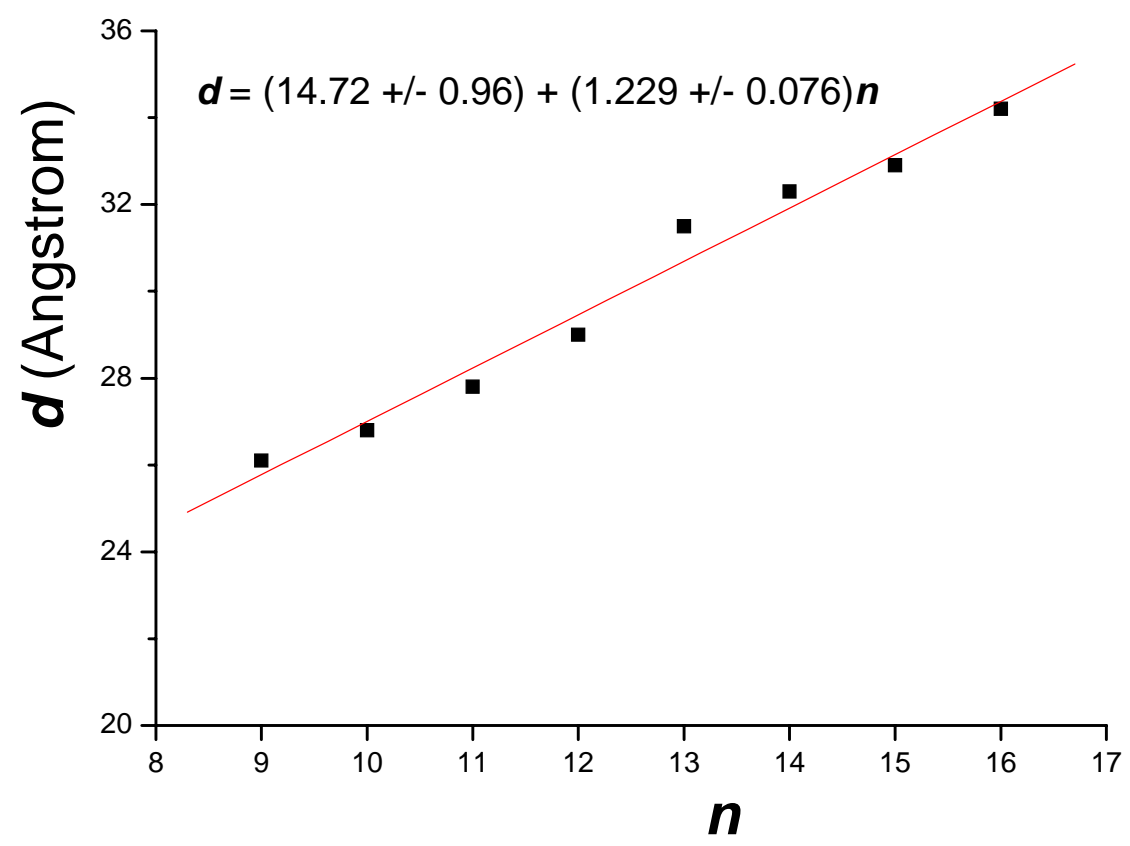

Figure S1. A plot of the lamellar period $\boldsymbol{d}$ versus the alkyl carbon number $\boldsymbol{n}$ for $\mathrm{C}_{n^{-}}$ Calix-OPP(1)-CN $(n=9-16)$

Linear Regression for Data1_B:

$\mathrm{Y}=\mathrm{A}+\mathrm{B} * \mathrm{X}$

\begin{tabular}{|c|c|c|}
\hline Parameter & Value & Error \\
\hline A & 14.71786 & 0.96089 \\
\hline B & 1.22857 & 0.07561 \\
\hline
\end{tabular}

\begin{tabular}{|c|c|c|}
\hline $\mathrm{R}$ & SD & $\mathrm{N}$ \\
\hline 0.98883 & 0.49002 & 8 \\
\hline
\end{tabular}


The XRD results for $\mathrm{C}_{8}$-Calix-OPP(1)-CN

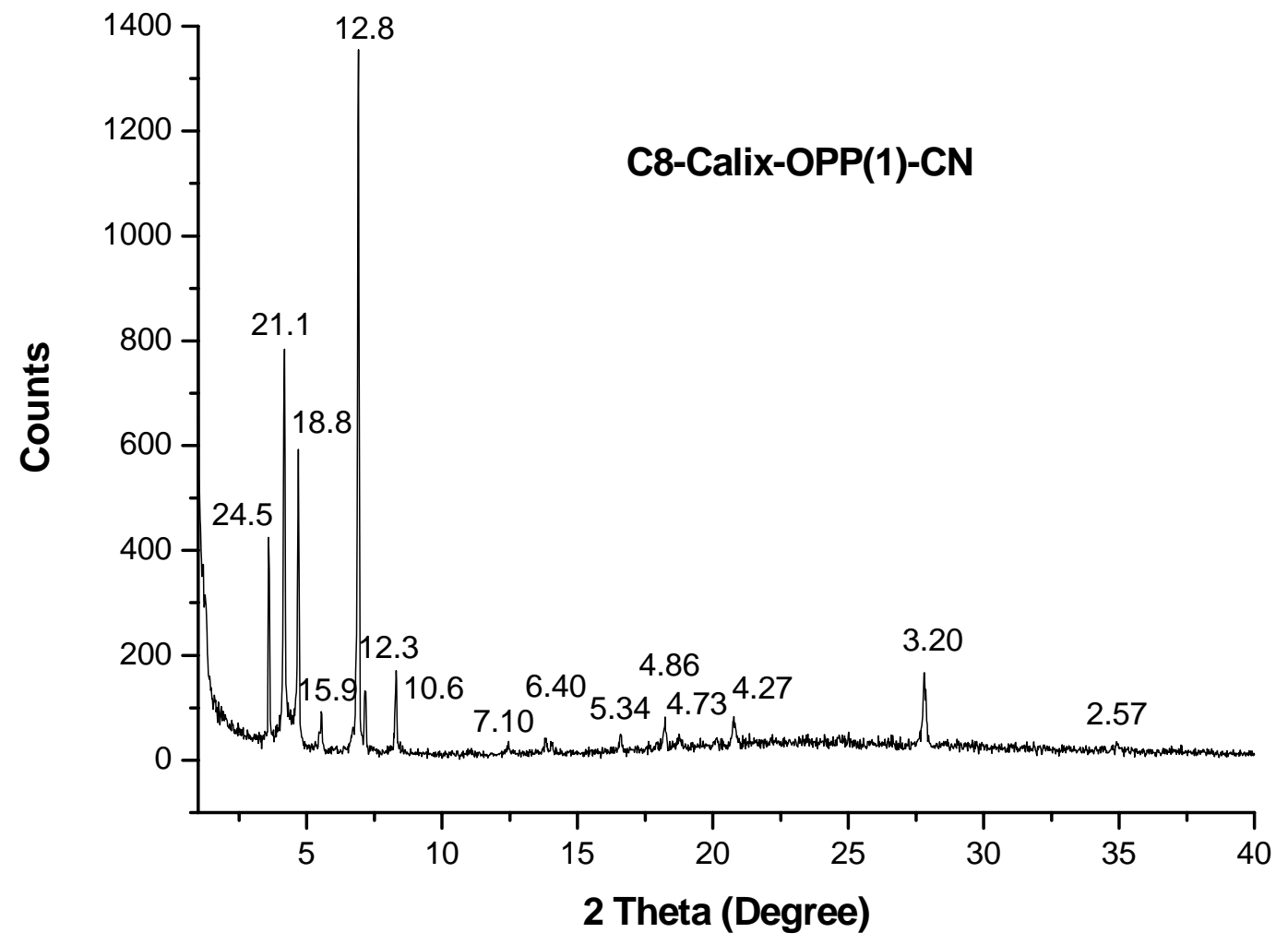

Figure S2. The XRD pattern of $\mathbf{C}_{8}$-Calix-OPP(1)-CN (the peak labels are in $\AA$, the sample experienced the same thermal history as other samples of the $\mathbf{C}_{n}$-Calix-OPP(1)-CN series)

Table S3. XRD data for $\mathrm{C}_{8}$-Calix-OPP(1)-CN crystallization

\begin{tabular}{|c|c|}
\hline $\begin{array}{c}\text { peak position } \\
\left(2 \theta /{ }^{\circ}\right)\end{array}$ & $\begin{array}{c}\text { corresponding } d \text { spacing }(\AA) \\
\text { (relative intensity, } \%)\end{array}$ \\
\hline 3.600 & $24.5(28.9)$ \\
\hline 4.180 & $21.1(58.1)$ \\
\hline 4.700 & $18.8(43.9)$ \\
\hline 5.540 & $15.9(7.08)$ \\
\hline 6.920 & $12.8(100)$ \\
\hline 7.160 & $12.3(11.0)$ \\
\hline 8.300 & $10.6(13.2)$ \\
\hline 12.46 & $7.10(2.42)$ \\
\hline 13.82 & $6.40(3.28)$ \\
\hline 14.06 & $6.29(2.76)$ \\
\hline 16.60 & $5.34(4.14)$ \\
\hline 18.24 & $4.86(5.95)$ \\
\hline 18.76 & $4.73(3.97)$ \\
\hline 20.78 & $4.27(6.56)$ \\
\hline 27.82 & $3.20(13.3)$ \\
\hline 34.92 & $2.57(2.93)$ \\
\hline & \\
\hline
\end{tabular}


The elevated temperature $\mathrm{XRD}$ results for $\mathrm{C}_{12}$-Calix-OPP(1)-CN and $\mathrm{C}_{16}-\mathrm{Calix}-\mathrm{OPP}(1)-\mathrm{CN}$

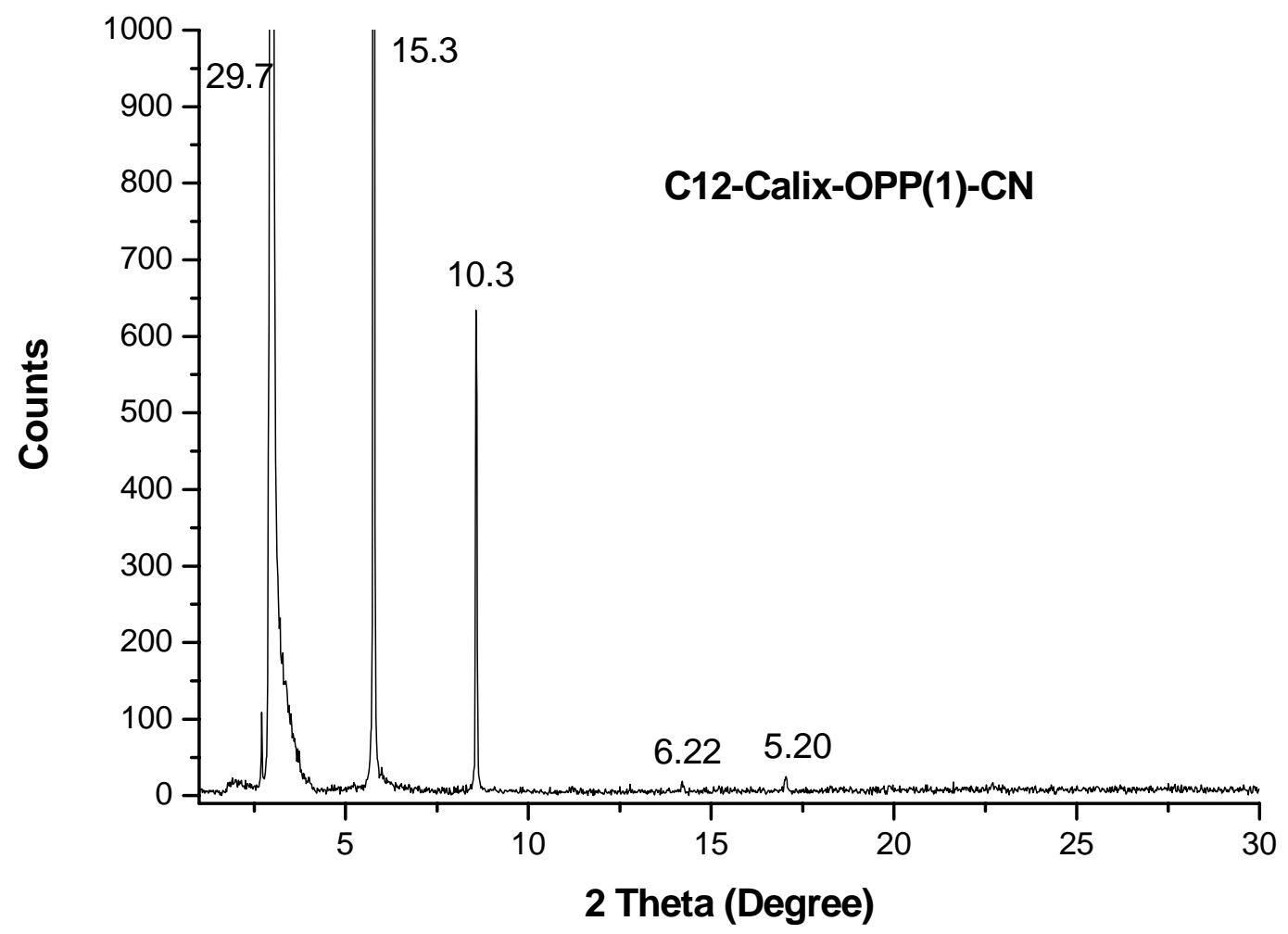

Figure S3. The elevated XRD pattern of $\mathbf{C}_{12}$-Calix-OPP(1)-CN at $160{ }^{\circ} \mathrm{C}$ (the peak labels are in $\AA$ )

Table S4. XRD data for $\mathrm{C}_{12}$-Calix-OPP(1)-CN at $160{ }^{\circ} \mathrm{C}$

\begin{tabular}{|c|c|c|c|}
\hline $\begin{array}{c}\text { peak position } \\
\left(2 \theta /{ }^{\circ}\right)\end{array}$ & $\begin{array}{c}\text { corresponding } d \text { spacing } \\
(\AA) \\
(\text { relative intensity, \%) }\end{array}$ & $\begin{array}{c}\text { relative ratio } \\
\text { of reciprocal } d\end{array}$ & $\begin{array}{c}\text { Proposed } \\
\text { indexing }\end{array}$ \\
\hline 2.703 & $32.7(0.20)$ & & \\
\hline 2.979 & $29.7(100)$ & 1.00 & $(001)$ \\
\hline 5.774 & $15.3(9.54)$ & 1.94 & $(002)$ \\
\hline 8.581 & $10.3(1.23)$ & 2.88 & $(003)$ \\
\hline 14.23 & $6.22(0.02)$ & 4.77 & $(005)$ \\
\hline 17.04 & $5.20(0.03)$ & 5.71 & $(006)$ \\
\hline & & & \\
\hline
\end{tabular}




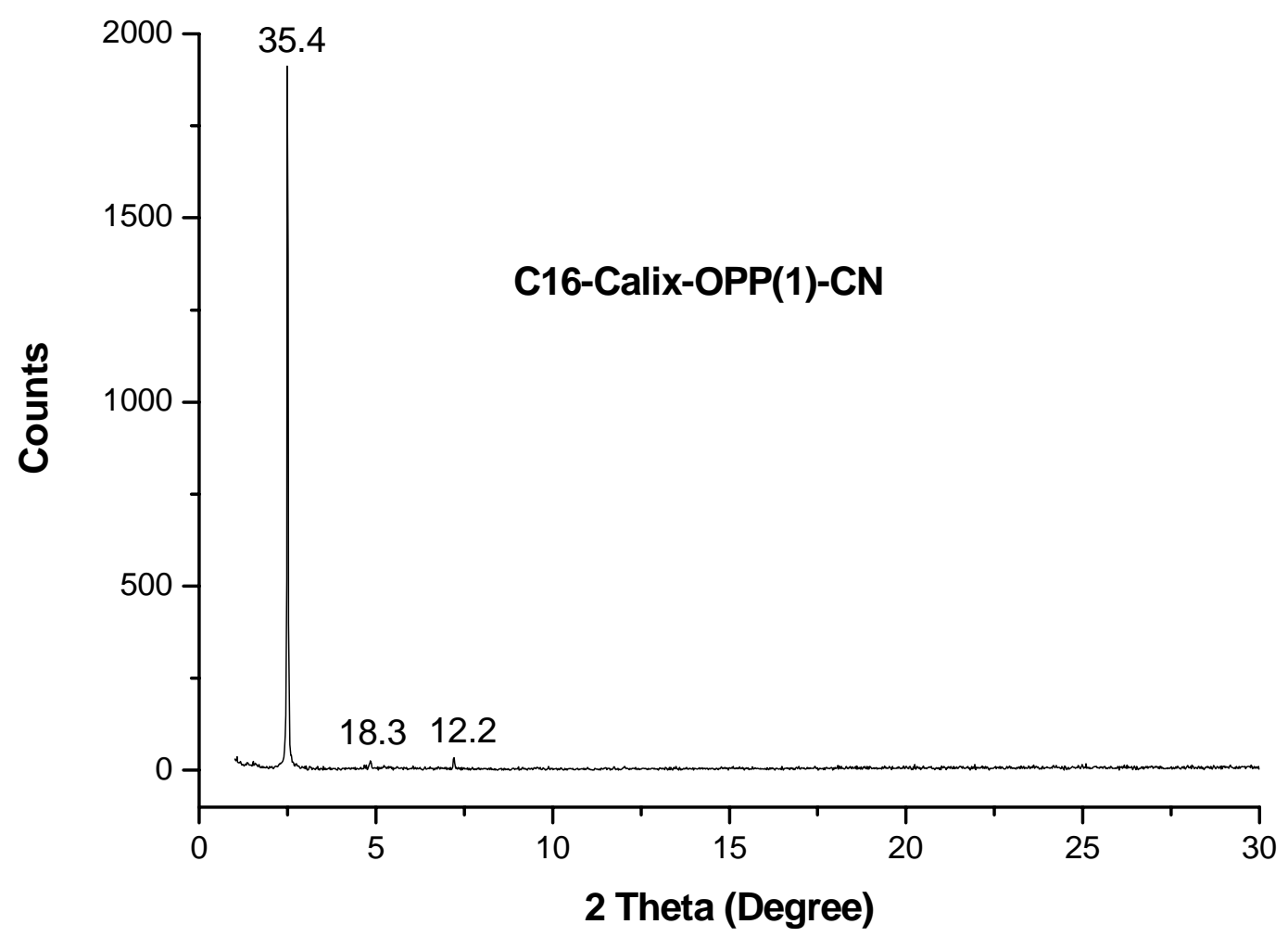

Figure S4. The elevated XRD pattern of $\mathbf{C}_{16}$-Calix-OPP(1)-CN at $130{ }^{\circ} \mathrm{C}$ (the peak labels are in $\AA$ )

Table S5. XRD data for $\mathrm{C}_{16}$-Calix-OPP(1)-CN at $130{ }^{\circ} \mathrm{C}$

\begin{tabular}{|c|c|c|c|}
\hline $\begin{array}{c}\text { peak position } \\
\left(2 \theta /{ }^{\circ}\right)\end{array}$ & $\begin{array}{c}\text { corresponding } d \text { spacing } \\
(\AA) \\
(\text { Relative intensity, \%) }\end{array}$ & $\begin{array}{c}\text { relative ratio of } \\
\text { reciprocal } d\end{array}$ & $\begin{array}{c}\text { proposed } \\
\text { indexing }\end{array}$ \\
\hline 2.499 & $35.4(100)$ & 1.00 & $(001)$ \\
\hline 4.836 & $18.3(0.94)$ & 1.93 & $(002)$ \\
\hline 7.235 & $12.2(0.56)$ & 2.90 & $(003)$ \\
\hline
\end{tabular}


The XRD results for the quenched LC samples of $\mathrm{C}_{9}$-Calix-OPP(2)-CH $\mathrm{CH}_{3}$ and $\mathrm{C}_{10}$-Calix$\mathrm{OPP}(2)-\mathrm{CH}_{3}$

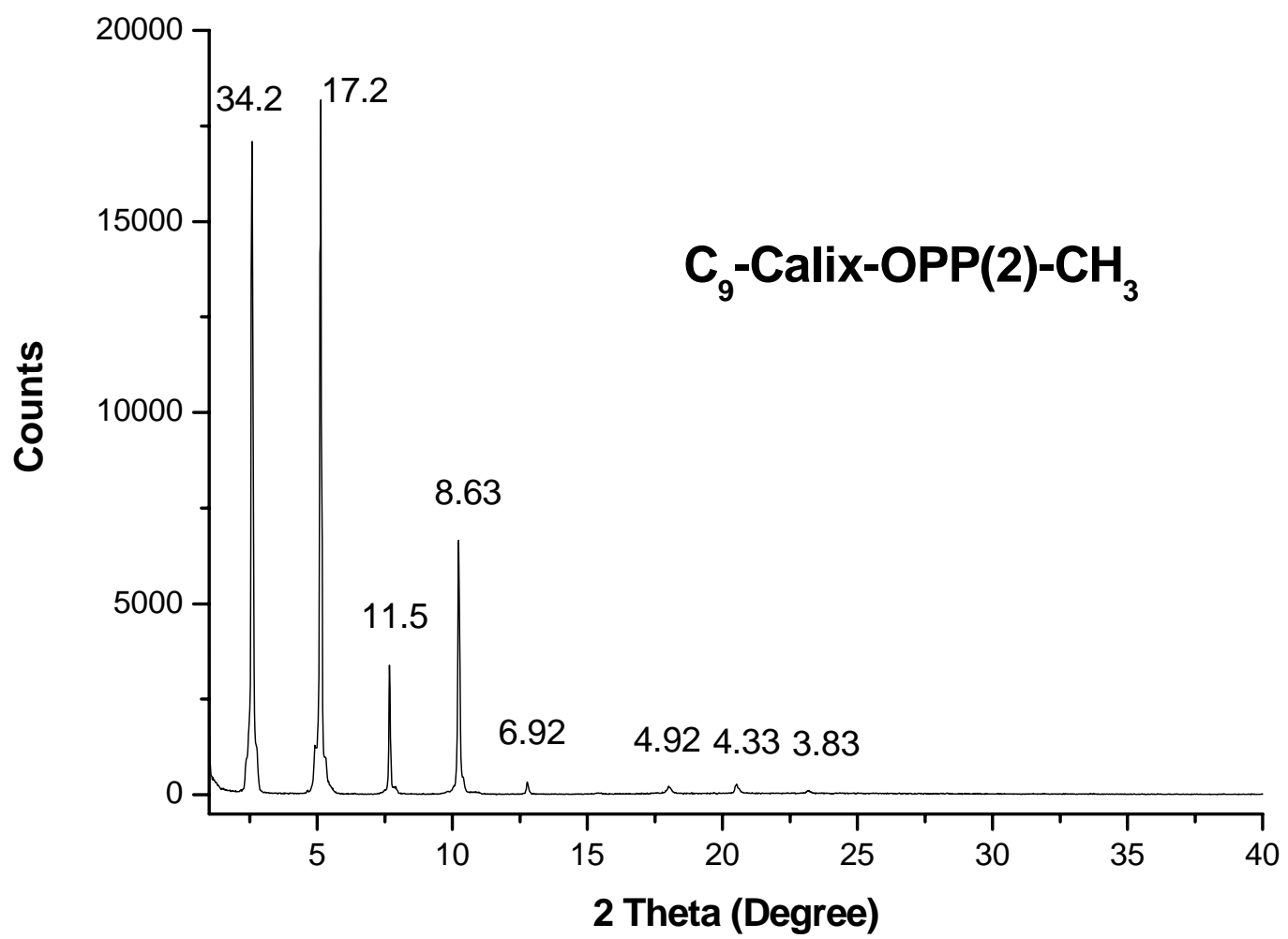

Figure S5. XRD pattern of $\mathrm{C}_{9}-\mathrm{Calix}-\mathrm{OPP}(2)-\mathrm{CH}_{3}$ quenched from $150{ }^{\circ} \mathrm{C}$ (the peak labels are in $\AA$ )

Table S6. XRD data for $\mathrm{C}_{9}$-Calix-OPP(2)- $\mathrm{CH}_{3}$

\begin{tabular}{|c|c|c|c|}
\hline $\begin{array}{c}\text { peak position } \\
\left(2 \theta /{ }^{\circ}\right)\end{array}$ & $\begin{array}{c}\text { corresponding } d \text { spacing } \\
(\AA) \\
\text { (relative intensity, \%) }\end{array}$ & $\begin{array}{c}\text { relative ratio of } \\
\text { reciprocal } d\end{array}$ & $\begin{array}{c}\text { proposed } \\
\text { indexing }\end{array}$ \\
\hline 2.580 & $34.2(100)$ & 1.00 & $(001)$ \\
\hline 5.120 & $17.2(99.0)$ & 1.99 & $(002)$ \\
\hline 7.680 & $11.5(19.0)$ & 2.97 & $(003)$ \\
\hline 10.24 & $8.63(39.5)$ & 3.96 & $(004)$ \\
\hline 12.78 & $6.92(2.08)$ & 4.94 & $(005)$ \\
\hline 18.02 & $4.92(1.34)$ & 6.95 & $(007)$ \\
\hline 20.52 & $4.33(1.75)$ & 7.90 & $(008)$ \\
\hline 23.20 & $3.83(0.69)$ & 8.93 & $(009)$ \\
\hline
\end{tabular}




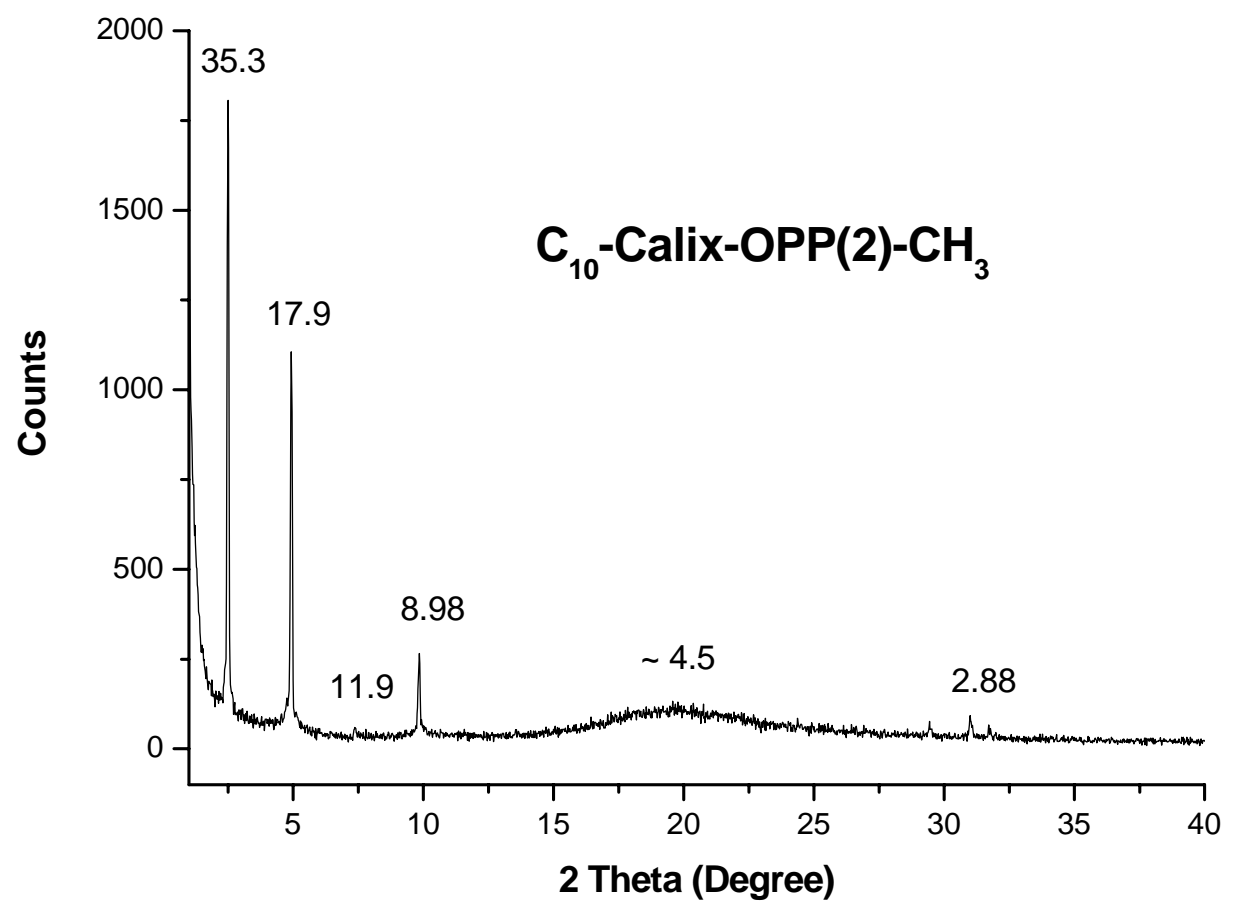

Figure S6. XRD pattern of $\mathrm{C}_{10}$-Calix-OPP(2)- $\mathrm{CH}_{3}$ quenched from $160{ }^{\circ} \mathrm{C}$ (the peak labels are in A)

Table S7. XRD data for $\mathrm{C}_{10}$-Calix-OPP(2)- $\mathrm{CH}_{3}$

\begin{tabular}{|c|c|c|c|}
\hline $\begin{array}{c}\text { peak position } \\
\left(2 \theta /{ }^{\circ}\right)\end{array}$ & $\begin{array}{c}\text { corresponding } d \text { spacing } \\
(\AA) \\
\text { (relative intensity, } \%)\end{array}$ & relative ratio & $\begin{array}{c}\text { proposed } \\
\text { indexing }\end{array}$ \\
\hline 2.500 & $35.3(100)$ & 1.00 & $(001)$ \\
\hline 4.940 & $17.9(61.8)$ & 1.97 & $(002)$ \\
\hline 7.420 & $11.9(2.17)$ & 2.97 & $(003)$ \\
\hline 9.840 & $8.98(14.4)$ & 3.93 & $(004)$ \\
\hline$\sim 20.0$ & $\sim 4.5($ diffuse) & & \\
\hline 31.00 & $2.88(5.22)$ & & \\
\hline
\end{tabular}

Only the XRD pattern of $\mathrm{C}_{10}$-Calix-OPP(2)-Me showing in Figure S6 displays a diffuse halo indicating some random liquid-like alkyl chains, no such remarkable halos are observed in all other $\mathrm{C}_{n}$-Calix-OPP $(m)$-X samples which could also imply a highly ordered smectic layered structures.. 
The relationship between the lamellar spacing $d$ and the molecular length $\mathrm{L}$ of $\mathrm{C}_{n}$-CalixOPP(m)-Xs

Table S8. $d \sim \mathrm{L}$ relationship for $\mathrm{C}_{n}$-Calix-OPP(m)-Xs*

\begin{tabular}{|c|c|c|c|}
\hline & $\begin{array}{c}\text { Experimental } \boldsymbol{d} \\
\text { spacing }\end{array}$ & Molecular length $\mathbf{L}$ & Ratio $\mathbf{d} / \mathbf{L}$ \\
\hline $\mathbf{C}_{\mathbf{9}}$-Calix-OPP(1)-CN & 26.1 & 20.6 & 1.3 \\
\hline $\mathbf{C}_{\mathbf{1 0}}$-Calix-OPP(1)-CN & 26.8 & 22.2 & 1.2 \\
\hline $\mathbf{C}_{\mathbf{1 1}}$-Calix-OPP(1)-CN & 27.8 & 22.5 & 1.2 \\
\hline $\mathbf{C}_{\mathbf{1 2}}$-Calix-OPP(1)-CN & 29.0 & 23.9 & 1.2 \\
\hline $\mathbf{C}_{\mathbf{1 3}}$-Calix-OPP(1)-CN & 31.5 & 25.1 & 1.3 \\
\hline $\mathbf{C}_{\mathbf{1 4}}$-Calix-OPP(1)-CN & 32.5 & 25.9 & 1.3 \\
\hline $\mathbf{C}_{\mathbf{1 5}}$-Calix-OPP(1)-CN & 32.9 & 27.1 & 1.2 \\
\hline $\mathbf{C}_{\mathbf{1 6}}$-Calix-OPP(1)-CN & 34.2 & 28.2 & 1.2 \\
\hline & & & 1.5 \\
\hline $\mathbf{C}_{\mathbf{9}}$-Calix-OPP(2)-CH & 34.2 & 23.3 & 1.4 \\
\hline $\mathbf{C}_{\mathbf{1 0}}$-Calix-OPP(2)-CH & 35.3 & 25.2 & \\
\hline
\end{tabular}

* The molecular length L's are measured as the height of the pinched cone conformation of $\mathrm{Cn}$ Calix-OPP $(m)-\mathrm{Xs}$ from the end methyl $\mathrm{C}$ of the lower rim alkyl to the $\mathrm{C}$ (for $-\mathrm{CH}_{3}$ ) or $\mathrm{N}$ (for$\mathrm{CN}$ ) of the upper $\operatorname{rim} \mathrm{X}$. 